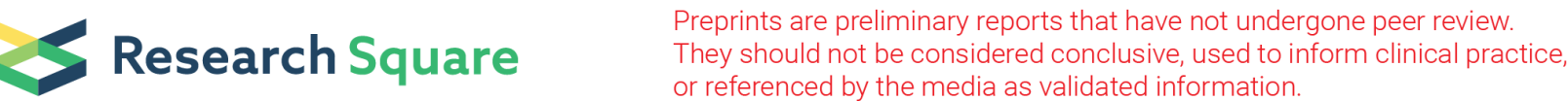

\section{Lipopolysaccharide-induced premature cervical ripening and inflammation in pregnant mice can be alleviated by nicotine}

Xinjia Han ( $\square$ hanxinjia@126.com )

Guangzhou Women and Children's Medical Center

Wen Ding

Guangzhou Women and Children's Medical Center

Baohua Lin

Guangzhou Women and Children's Medical Center

Juanhua Chen

Guangzhou Women and Children's Medical Center

Tiansong Zhang

Guangzhou Women and Children's Medical Center

Yumian Lai

Guangzhou Women and Children's Medical Center

Jinying Yang

Guangzhou Women and Children's Medical Center

\section{Research}

Keywords: nicotine, a7nAChR, cervical ripening, macrophage polarization, inflammation

Posted Date: April 30th, 2020

DOl: https://doi.org/10.21203/rs.3.rs-24975/v1

License: (c) (i) This work is licensed under a Creative Commons Attribution 4.0 International License.

Read Full License 


\section{Abstract \\ Background}

Clinical treatment of preterm birth (PTB) is usually dependent on inhibiting uterine contractions. Such therapy is only effective in the short-term and cannot fundamentally low the incidence of PTB. Premature cervical maturation is another important cause of PTB; it is associated with invasion of macrophages, which produce proinflammatory cytokines. We previously found that activation of the alpha-7 nicotinic acetylcholine receptor ( $\mathrm{a} 7 \mathrm{nAChR})$ by nicotine alleviates the systemic inflammatory response in murine models of lipopolysaccharide (LPS)-induced PTB, but the underlying mechanisms remain unclear.

\section{Methods}

Cervical tissues were collected from women in preterm and term labor. An animal model of PTB was established by administering $25 \mu \mathrm{g} / \mathrm{kg}$ lipopolysaccharide (LPS) to normal pregnant C57BL/ 6 mice on gestational day 16 (GD16). PTB animals received $1 \mathrm{mg} / \mathrm{kg}$ of a7nAChR agonist nicotine or nicotine combined with $1 \mu \mathrm{g} / \mathrm{kg}$ a7nAChR antagonist a-bungarotoxin (a-BGT) from GD14 to GD17. The gestational age, the rates of preterm birth and the survival rate of pups were recorded. Immunofluorescence staining were utilized for the quantification of a7nAChR expression on cervical macrophages and changes in macrophage polarization, RT-PCR was used to determine the mRNA levels of M1 and M2 macrophage biomarkers. Elisa assay was used to detect changes in fetal inflammation. Immunofluorescence staining and western blotting were used to investigate the signaling pathways underlying nicotine's promoting LPS-induced conversion of M1 macrophage to M2 in the cervix of PTB animals.

\section{Results}

a7nAChR expression on cervical macrophages and cervical collagen content was decreased in PTB patients and murine models of LPS-induced PTB. The number of M1 cervical macrophages, other proinflammatory mediators in the cervix and fetal inflammation was unregulated in pregnant mice following LPS administration. Nicotine treatment reduced the rate of PTB, decreased fetal inflammation, improved maternal and neonatal outcome; nicotine also increased polarization of cervical macrophages toward the anti-inflammatory M2 phenotype possibly by suppressing activation of the MAP kinases JNK and ERK1/2 and nuclear translocation of NF-KB and rescuing the inhibited JAK2/STAT3 and PI3K/AKT pathways. The effects of nicotine were reversed by the selective a7nAChR antagonist a-bungarotoxin (aBGT).

\section{Conclusions}


Our findings indicate that nicotine prevents LPS-induced cervical ripening and inflammatory response by inducing M2 macrophage polarization, and nicotine may serve as potential anti-premature cervical maturation agents for treatment of PTB.

\section{Background}

Clinically, drugs that inhibit uterine contractions are the mainstay treatment for preterm birth (PTB) [1]. Although administration of tocolytic agents such as $\beta 2$-adrenergic receptors, oxytocin-receptor antagonists, and prostaglandin synthetase inhibitors can inhibit uterine contraction within $48 \mathrm{hrs}$, they have not fundamentally decreased the incidence of PTB $[2,3]$. Consequently, there is an unmet need to further elucidate the pathophysiology of PTB and find more effective therapeutic targets.

Evidence suggests that premature cervical maturation is an important cause of PTB, and changes in collagen content reflect cervical maturity. Collagen level in the cervical tissue is $60 \%$ lower in pregnancy than in nonpregnancy and significantly lower in PTB patients and murine models of PTB than in normal pregnancy [4]. Increased secretion of collagenases (matrix metallopeptidase [MMP]-2, MMP-9) in the cervical tissues [5], cervicovaginal fluid [6], amnion and chorion [7] are responsible for cervical ripening and fetal membrane rupture at term birth, possibly by prompting breakdown of extracellular matrix (ECM) components consisting predominantly of collagens. An imbalance in the expression of MMPs and their inhibitors (TIMPs) is found in the preterm placenta and amniotic membranes [8, 9]. Based on these findings, we propose that regulation of collagen content and collagenase level in the cervix represent key factors in the treatment of PTB.

Cervical ripening in normal pregnancy is an inflammatory process [10] that involves an increase in the expression of inflammatory cytokines. In PTB, the inflammatory response in the cervix is exaggerated [11]. Watari et al. reported that increased expression of inflammatory cytokines was associated with increased MMPs expression in smooth muscle cells from human cervix $[12,13]$. In other studies, TNF-a and IL-33 induced monocytes/macrophages to secrete MMP-9 $[14,15]$. These data imply that excessive inflammation in the cervix has potential to upregulate MMP-9 expression, which promotes the degradation of collagens to cause premature cervical maturation.

Under different physiological and pathological environments, macrophages can be polarized into two states, M1 and M2 phenotypes, which have disparate roles in tissue remodeling [16]. M1 macrophages secrete inflammatory factors and MMPs to degrade the ECM. M2 macrophages secrete anti-inflammatory factors, including TGF- $\beta$ and IGF-1, to promote the secretion of TIMPs and inhibit ECM degradation [17]. Numerous studies have demonstrated that increasing the proportion of M2/M1 macrophages improves the inflammatory microenvironment and inhibits collagen degradation to promote tissue repair after injury [18-20]. Cervical ripening is a process of ECM remodeling. A previous investigation showed a significant infiltration of macrophages into the cervix of normal pregnant women during cervical ripening in the third trimester, and that CD68-stained macrophages increased to an equivalent extent in cervical subepithelium and stroma from women in term labor, preterm labor, and not in labor at term [21]. Another 
report showed the number of activated macrophages involved in MMP induction peaked on the 18th day of gestation in the cervix of pregnant mice [22]. These data suggest that macrophage infiltration participates in the process of cervical ripening. However, changes in the polarization state of macrophages in preterm labor remain to be elucidated.

a7nAChR is widely expressed on macrophages. In clinical trials, activation of a7nAChR was used to treat inflammatory diseases such as enteritis and rheumatoid arthritis [23], and inhibition of a7nAChR was used to attenuate tissue fibrosis related to inflammation [24]. The regulatory role of a7nAChR in tissue fibrosis and tissue repair has been related to changes in collagen content [25]. Our previous studies showed that an a7nAChR agonist (nicotine) prolonged normal gestation and inhibited collagen degradation and cervical ripening in pregnant murine. Our further studies revealed that the serum levels of inflammatory cytokines, such as TNF-a and IL-6, were up-regulated in animal models of LPS-induced inflammation, and nicotine could reduce the serum levels of inflammatory cytokines [26]. However, the function and mechanisms of nicotine on macrophages in the cervix in PTB have not been clearly defined.

In the present study, we examined the expression level of nicotine receptor ( $\mathrm{a} 7 \mathrm{nAChR}$ ) on cervical macrophages in PTB patients and LPS-induced inflammatory animal models, investigated the role of nicotine in PTB in terms of collagen degradation, local inflammation of cervix, fetal inflammation, cervical macrophage polarization and the related downstream signaling pathway of a7nAChR. Our results show that the protective effects of nicotine in pregnant mice following LPS administration are related to promote cervical macrophage M2 polarization to reduce inflammation and collagen degradation.

\section{Methods}

\section{Cervical Biopsies}

Fresh cervical tissue specimens were obtained from women in preterm $(n=10)$ and term labor $(n=12)$. Biopsies were taken from the anterior cervical lip immediately after vaginal delivery or before caesarean section. Women with cervical dysfunction, premature rupture of fetal membranes, placenta previa, hydramnios, history of late abortion or uterine deformity were excluded from this study.

After biopsy, tissues were quickly washed with $0.9 \%$ physiological saline, and fixed with $4 \%$ paraformaldehyde in $0.01 \mathrm{M}$ phosphate buffer (PBS) for $1-2$ days at $4{ }^{\circ} \mathrm{C}$. Cervical tissue was paraffinembedded, sectioned to a $4 \mu \mathrm{m}$ thickness, and stained in Masson's trichrome and for immunohistochemistry analysis.

This study was approved by the Ethics Committee of the Guangzhou Women and Children Medical Center (No. 2018041701 and No. 201922200), and all included patients provided written informed consent.

\section{Animals}


Adult C57BL/6 mice (8-12 weeks) were purchased from Guangdong Medical Laboratory Animal Center, Guangzhou, China. The mice were acclimatized to the animal facility for one week before the study. Mice were bred in an environment with free access to food and water and with a $12 \mathrm{~h}$ light- $12 \mathrm{~h}$ dark cycle. Recipient female mice were mated to males. Vaginal plug observation indicated gestational day 1(GD1).

Pregnant mice were randomized into four groups: normal pregnant controls $(P, n=18)$; LPS, (pregnant mice treated with LPS, $n=18$ ); LPS $+N$ (pregnant mice treated with LPS and nicotine, $n=18$ ); $L P S+N+A$, (pregnant mice treated with LPS, nicotine and the $a 7 n A c h R$ antagonist, $a-B G T, n=18$ ).

All animal protocols were approved by the Committee on the Ethics of Animal Experiments of Guangzhou Medical University (Permit Number: 2012-50).

\section{Animal models of premature birth and treatment protocols}

According to methods described in studies from Nardhy et al. [27, 28], LPS (strain Escherichia coli 0111:B4, cat\# L4130) and nicotine hydrogen tartrate (a7nAchR agonist) (cat\# BP822) were purchased from Sigma-Aldrich (St. Louis, MO, USA). LPS and nicotine were each dissolved in $0.9 \%$ physiological saline for a $1.0 \mathrm{mg} / \mathrm{ml}$ stock solution, and the $\mathrm{pH}$ of the nicotine solution was adjusted to $7.1-7.3$ using $\mathrm{NaOH} .1 .0 \mathrm{mg} / \mathrm{ml}$ stock solutions were stored frozen in aliquots at $-20^{\circ} \mathrm{C}$, and thawed and diluted for use. 1 mg a-BGT (cat\# ZX0001, Shanghai BOYAO Biological Technology Co., Ltd; China) was dissolved in $10 \mathrm{ml} 0.9 \%$ physiological saline, stored frozen in aliquots at $-20^{\circ} \mathrm{C}$, and thawed and diluted to $0.1 \mu \mathrm{g} / \mathrm{ml}$ for use.

On GD16, $25 \mu \mathrm{g} / \mathrm{kg}$ LPS was administered by intraperitoneal injection to pregnant mice to establish a model of PTB. From GD14 to GD17, nicotine exposed PTB mice were administered $1 \mathrm{mg} / \mathrm{kg}$ nicotine daily in two subcutaneous injections at a 6-hour interval; on GD16, nicotine was administered 40 minutes before LPS. From GD14 to GD17, nicotine exposed PTB mice treated with a-BGT were administered $1 \mu \mathrm{g} / \mathrm{kg}$ a-BGT daily in two intraperitoneal injections at a 6-hour interval; a-BGT was administered 30 minutes before nicotine.

Mice were scarified on GD 18. Cervical tissue was harvested immediately under anesthesia. Cervical tissue was paraffin-embedded, sectioned to a $4 \mu \mathrm{m}$ thickness, and stained in Masson's trichrome and for immunohistochemistry analysis, or frozen in liquid nitrogen and stored at $-80^{\circ} \mathrm{C}$.

\section{Masson's trichrome staining}

Paraffin sections were deparaffinized and rehydrated, stained in iron hematoxylin dye (cat\# G1006, Servicebio, Wuhan, China), Biebrich scarlet-acid fuchsin solution(cat\# G1005-3, Servicebio) and phosphomolybdic-phosphotungstic acid solution (cat\# G1005-4, Servicebio), and mounted in glycerol jelly mounting medium (cat\# C0187, Beyotime Biotechnology). Images were captured under a Leica DM4B microscope (Wetzlar, Germany). Cervical collagen content was analyzed semi-quantitatively as previously described $[29,30]$. Image Pro Plus 6.0 software was used to determine staining intensity, 
calculated as the integrated optical density. A total of 30 sections ( 5 sections/animal, 6 animals/group) were used for imaging.

\section{Immunohistochemistry}

Paraffin sections were dewaxed and rehydrated, antigen retrieval was performed, sections were incubated in blocking buffer ( $3 \%$ bovine serum albumin in $0.03 \%$ Triton diluted with $0.01 \mathrm{M} \mathrm{PBS}$ ) for $2 \mathrm{~h}$ at room temperature. Subsequently, sections were incubated with primary antibodies for $20 \mathrm{~h}$ at $4{ }^{\circ} \mathrm{C}$.

For single immunofluorescence staining, antibodies were collagen type I (rabbit, 1:100, cat\# AF7001),collagen type III (rabbit, 1:100, cat\# AF0136), TIMP-1 (rabbit, 1:100, cat\# AF7007), and MMP-9 (rabbit, 1:100 cat\# AF5228, Affinity Biosciences, Cincinnati, OH, USA). For double immunofluorescence staining, antibodies were a7nAChR (rabbit, 1:100, cat\# ab10096), CD68 (mouse,1:50, cat\# ab955), CD11b (rat, 1:100, cat\# ab8878),CD86 (rabbit, 1:200, cat\# ab125212),CD163(rabbit, 1:200, cat\# ab182422), iNOS (rabbit, 1:50, cat\# ab15323, Abcam, Cambridge, MA, USA); Arg-1, (rabbit, 1:50, cat\# 93668) (Cell Signaling Technology, Danvers, MA, USA); TNF- $a$, (rabbit, 1:100, cat\# bs-10802R), IL-1 $\beta$ (rabbit, 1:100, cat\# bs0812R), and NF-KB p65 (rabbit, 1:100, cat\#ab16502). After incubation, sections were washed in $0.01 \mathrm{M}$ PBS and incubated with a secondary antibody for $3 \mathrm{~h}$ at room temperature. Secondary antibodies were goat anti-rabbit (1:500, cat\# ab150081, Alexa Fluor 488), goat anti-rat, (1:500, cat\# ab150165, Alexa Fluor 488), goat anti-mouse (1:500, Cat\# ab150117, Alexa Fluor 488), donkey anti-goat (1:500, cat\# ab150129, Alexa Fluor 488), and goat anti-rabbit (1:500, cat\# ab150088, Alexa Fluor 594). Sections were washed with $0.01 \mathrm{M}$ PBS and mounted with fluoroshield with DAPI mounting medium (cat\# ab104139, Abcam). Sections were photographed with a fluorescence microscope (DM4B, Leica, Wetzlar, Germany).

For single immunofluorescence staining, $\mathrm{NIH}$ Image $\mathrm{J}$ software was used to quantify fluorescence intensity (FD) of the stained areas (SA) relative to the background. A total of 28 sections (4 sections/animal, 7 animals/group) were analyzed. Images were obtained under the same light exposure time and magnification (X10 and X20).

For double immunofluorescence staining, NIH ImageJ software was used to quantify the number of CD11b positive cells in a blinded fashion. A total of 36 sections ( 6 sections/animal, 6 animals/group) were analyzed. Values were expressed as percent change from the normal pregnant controls $(P)$.

\section{Real-time PCR}

On GD18, the cervical tissues were dissected out from the anesthetized animals and stored at $-80^{\circ} \mathrm{C}$ .Total RNA was extracted from the cervical tissues using the RNeasy mini kit (Qiagen, cat\#74104, Germany). A quantitect Reverse Transcription Kit (Qiagen, cat\# 205310, Germany) was used to generate cDNA. The cDNA was used as template for quantitative RT-PCR, which was performed using the SYBR Green qPCR SuperMix kit (invitrogen by Thermo Fisher Scientific, cat\#11784200, CA, USA) and the ABI PRISM ${ }^{\circledR} 7500$ Sequence Detection System (invitrogen by Thermo Fisher Scientific, CA, USA). Samples were run in triplicate. Primer sequences were: TNF-a, 5'-CTACTCCCAGGTTCTCTTCAA - $3^{\prime}$ (forward) and 5'-GCTGACTTTCTCC -TGGTATGA-3'(reverse); IL-1 $\beta$, 5'-GTG C -TGTCTGACCCATGTGA - 3' (forward) and 
5'-CACAGGGATTTTGTCGTTGCT - 3' (reverse); CCL2, 5'-CAGGTCTCTGTC -ACGCTTCT - 3' (forward) and 5'-CTGC -TGCTGGTGATTCTCTT - 3' (reverse); CXCL10,5'- ACCCAGGGCCATAG GAAA -A -3' (forward) and 5'-CCGGATTC -AGACACCTCTTC-3' (reverse); CD86, 5'- TTGCAGGTCCCAGTTCACTT-3' (forward) and 5'CTGTCCTGCTTGGAC- TCACA-3' (reverse); iNOS, 5'- GAGCAAAAAAGGGCAACAC-3' (forward) and 5'CGCACTTCTGTC TC TC -CAAA-3' (reverse); ARG-1,5'- TTGGCAAAGTGATG -GAAGAG-3' (forward) and 5'GTAAGATAGGCCTCCCACAA-3' (reverse); IL-10, 5'-TCTACAAGGC -CATGAATGAG-3' (forward) and 5'GAGAGAGGTACAA AC -GAGG-3' (reverse); CD163, 5'- CCTTCCTCTTGTGGACTCTGA-3' (forward) and 5'TTAAATGC CA -ACCCGAGGAT-3' (reverse); CD206, 5’ GCTACCAGGAA- GTCCATCAT-3' (forward) and 5'CATAGGGCCACCACTGATTA-3' (reverse). All procedures were carried out according to the manufacturer's instructions. To ensure equal loading, $\beta$-actin (housekeeping gene) was used as an internal control.

\section{Enzyme-linked immunosorbent assay}

On GD18, the amniotic fluid was drawn out from the anesthetized animals using a 26-G needle and centrifuged at $1,000 \times \mathrm{g}$ for $15 \mathrm{~min}$ at $4{ }^{\circ} \mathrm{C}$. The supernatant was separated and stored at $-80{ }^{\circ} \mathrm{C}$. Chemokine/cytokine concentrations (IFN- $\gamma$, IL-1ß, IL-4, IL-6, IL-10, IL-13, CXCL10, CCL-2, CCL-5, TNF-a) were measured using commercial enzyme-linked immunosorbent assay (ELISA) kits (Solarbio LIFE SCIENCES, Beijing, China), according to the manufacturer's instructions. All samples were run in duplicate.

\section{Western Blotting}

Cervical tissues were digested in RIPA Lysis Buffer (cat\# P0013C, Beyotime Biotechnology, Shanghai, China) containingprotease inhibitors (1:100) (PMSF, cat\# ST506, Beyotime Biotechnology). Tissues were homogenized and centrifuged at $15000 \mathrm{rpm}$ for $10 \mathrm{~min}$ at $4{ }^{\circ} \mathrm{C}$. Protein in the supernatant was quantified using the BCA Protein Assay Kit (cat\# P0012, Beyotime Biotechnology). Proteins were separated by polyacrylamide gel electrophoresis (10\% SDS-PAGE) and transferred to $0.45 \mu \mathrm{m}$ and $0.22 \mu \mathrm{m}$ polyvinylidene difluoride (PVDF) membranes. Membranes were rinsed in 0.01M TBST and incubated in $5 \%$ skim milk powder in 0.01M TBST for $2 \mathrm{~h}$ at room temperature. Membranes were washed in 0.01M TBST and incubated with primary antibodies overnight at $4{ }^{\circ} \mathrm{C}$. Primary antibodies were: JNK1 + JNK2 + JNK3(rabbit, 1:1000; cat\# bs-2592R), p-JNK1 + JNK2 + JNK3 (rabbit, 1:1000; cat\# bs-4163R), ERK1/2 (mouse, 1:600, cat\# bsm-33337M), p-ERK1/2 (rabbit, 1:700; cat\# bs-3016R), JAK2 (rabbit, 1:800, cat\# bs23003R), p-JAK2 (rabbit, 1:500, cat\# bsm-52171R), SAT3 (rabbit, 1:500, cat\# bsm-33218M), p-SAT3 (rabbit, 1:500, cat\# bs-1658R), AKT1(rabbit, 1:500, cat\# bs-0115R), actin (rabbit, 1:2000; cat\# bs-10966R, Bioss Biotechnology, Beijing, China); p-AKT1 (rabbit, 1:800, cat\# 9018S, CST); and NF-kB p65 (rabbit, 1:800, cat\# ab16502, Abcam).Membranes were rinsed in 0.01M TBST and incubated with secondary antibodies for $2 \mathrm{~h}$ at room temperature. Membranes were washed with TBST and proteins were detected with the ECL Western blotting Detection Kit (WBKLS001000; Millipore, Burlington, MA, USA) and exposed on a gel imaging system (ChemiDoc XRS; Bio-rad, Hercules, CA, USA). NIH ImageJ software was used for densitometric analysis.

\section{Statistical Analysis}


Statistical analyses were conducted with SPSS v17.0 (SPSS, Chicago, IL, USA). For the immunoreactivity data, Masson staining results and the protein expression by western blotting, between-group differences were compared using the one-way analysis of variance (ANOVA); a least significant difference (LSD) post hoc test when groups had the same variance or Dunnett's test when variances were not equal;

data are expressed as the mean \pm SEM. For rates of preterm labor, gestational age, pup survival rate, mRNA expression and chemokine/cytokine concentrations, between-group differences were compared using the Mann-Whitney $U$ test. A $p$ value $<0.05$ was considered significant.

\section{Results}

Nicotine significantly decreased the frequency of preterm labor and prolonged the gestation in a pregnant mouse model of LPS induced inflammation

Pregnant mice injected with LPS had a higher frequency of preterm labor (LPS vs. P, $66.7 \%$ vs. $0 \%, p=0.003$, Figure $1 \mathrm{~A}$ ) and a shorter gestation than normal pregnant controls (LPS vs. $P, 18.6 \pm 0.16$ vs. $18.6 \pm 0.11$ days, $p=0.002$, Figure $1 B$ ). Nicotine treatment decreased the PTB rate to $12.5 \%$ and prolonged gestation to $19.4 \pm 0.11$ days. $\alpha-B G T$, an inhibitor of a7nAChR, reversed the beneficial effects of nicotine (Figure 1A and 1B).

\section{Nicotine significantly improved fetal outcomes}

The live birth rate was decreased in pregnant mice injected with LPS compared to normal pregnant controls (LPS vs. P, $31.6 \%$ vs. $100 \%, p=0.001$, Figure 1C). Nicotine treatment markedly increased the live birth rate to $77.5 \%$ in mice injected with LPS. $\alpha$-BGT blocked the nicotine effect to some extent (LPS $+N+A$ vs. LPS $+N, 39.3 \%$ vs. $77.5 \%, p=0.029$, Figure 1C).

$\alpha 7 n A c h R$ expression on cervical macrophages was decreased in PTB patients and pregnant mice injected with LPS

Patient information was provided in Table 1. The gestational age at delivery in PTL group was lower than that in TL group $(\mathrm{p}<0.01)$; the number of previous preterm deliveries seemed more in PTL compared to TL groups (PTL vs. TL, 3 vs. 0). 
Table 1. Patient characteristics for immunohistochemistry and Masson staining study in Cervix Biopsies from women who were PTL and TL.

\begin{tabular}{|c|c|c|c|c|}
\hline & Age(years) & $\begin{array}{l}\text { Previous } \\
\text { PTL }\end{array}$ & $\begin{array}{l}\text { Previous cervical } \\
\text { surgery }\end{array}$ & $\begin{array}{l}\text { Gestational } \\
\text { age(weeks) }\end{array}$ \\
\hline $\mathrm{TL}(\mathrm{n}=12)$ & $29(20-38)$ & 0 & 0 & $39(38-40)$ \\
\hline $\begin{array}{l}\text { PTL } \\
(n=10)\end{array}$ & $28(20-36)$ & 3 & 0 & $32(28-35){ }^{* *}$ \\
\hline
\end{tabular}

PTL, spontaneous preterm labor through vaginal delivery; TL, term labor through vaginal delivery. The data are expressed as median and analyzed with the nonparametric two-tailed t test. ${ }^{* *} \mathrm{P}<0.01$, compared to TL.

To investigate the possible role of $\alpha 7 \mathrm{nAchR}$ on cervical macrophages in $\mathrm{PTB} \square$ we analyzed the expression level of $\alpha 7 \mathrm{nAchR}$ on cervical macrophages from PTB patients and pregnant mice with LPS injection by double immunofluorescence staining.CD68 and CD11b were used as markers of cervical macrophages for humans and mice, respectively. Findings showed $\alpha$ nAchR immunoreactivity on cervical macrophages from PTB patients (Figure 2A) and pregnant mice following LPS administration (Figure 2B) were significantly decreased compared to normal pregnant controls. Treatment of PTB mice with nicotine rescued $\alpha 7 n A c h R$ expression on cervical macrophages, but that protection was reversed by treatment with $\alpha$-BGT (Figure 2B).

$\alpha 7 n A c h R$ activation by nicotine has the potential to inhibit premature cervical ripening in PTB mice

Changes in cervical collagen play a key role in the regulation of cervical ripening. To investigate the regulatory function of $\alpha 7 \mathrm{nAchR}$ in cervical ripening, we analyzed the collagen content of cervical tissue from PTB patients and mice by Masson's trichrome and immunofluorescence staining. Consistently, there was a substantial decrease in the number of blue stained collagenous fibers in cervical tissues from PTB patients (Figure 3A) and 
mice (Figure 3B), and collagen I and collagen III immunoreactivities were reduced in cervical tissues from PTB model compared to normal pregnant mice (Figure 3C and 3D). Nicotine treatment significantly increased the number of blue stained fibers in cervical tissue from PTB (Figure 3B) and the fluorescence intensity of collagen I and collagen III (Figure 3C and 3D).These effects were blocked by treatment with $\alpha-B G T$. These data suggest that $\alpha 7 \mathrm{nAchR}$ activation attenuated the decrease in collagen content in the cervix of PTB mice, and imply that $\alpha 7 n A c h R$ activation by nicotine has potential to inhibit premature cervical ripening.

Nicotine treatment inhibited MMP-9 expression in cervical tissues from PTB mice

Degradation of cervical collagens depends on the relative levels of MMP-9 and its inhibitor TIMP-1. To investigate whether the increase in cervical collagen content following $\alpha 7 n A c h R$ activation in PTB was related to decreased expression of MMP-9, we analyzed the immunoreactivity of MMP-9 and its inhibitor (TIMP-1) in cervical tissues from PTB mice. Findings showed that the MMP-9 immunoreactivity was significantly increased (Figure 4A) while TIMP-1 immunoreactivity (Figure 4B) was significantly decreased in cervical tissues from PTB mice compared to normal pregnant controls. Nicotine treatment remarkably reduced the immunoreactivity of MMP-9 but enhanced the immunoreactivity of TIMP-1; to some extent, these changes were reversed by treatment with $\alpha$-BGT. These data indicate that collagen content in cervical tissues decreased in association with an increase in the expression of MMP-9 in PTB mice. Nicotine treatment attenuated the increase in MMP-9 expression and collagen degradation, and may prevent premature cervical ripening.

Macrophage M1 polarization in cervical tissues from PTB mice was inhibited by nicotine

We previously found that systemic inflammation had a role in promoting premature cervical ripening in PTB [26]. Macrophage infiltration into the cervix is significantly increased during PTB [21]. Macrophages are polarized into different inflammatory phenotypes, which have varying pathological consequences. To investigate whether changes in cervical collagen content and MMP-9 expression in PTB following $\alpha 7 \mathrm{nAchR}$ activation are associated with reduced local inflammation in the cervix, we identified M1 
and M2 macrophages in cervical tissues by immunofluorescence staining. M1 macrophages were identified using combinations of CD86, iNOS, inflammatory cytokine (TNF- $\alpha$, IL-1 $\beta$ ), and CD11b (macrophage marker) antibodies. M2 macrophages were identified using combinations of CD163, Arg-1, and CD11b antibodies. Findings showed significantly more M1 macrophages (Figure 5A) and significantly less M2 macrophages (Figure 5B) were present in cervical tissues from PTB mice compared to pregnant controls. Significantly more M1 surface (iNOS, Figure 6A) and phenotypic (inflammatory cytokines: TNF- $\alpha$ and IL-1 $\beta$, Figure 7) markers and less M2 surface markers (Arg-1, Figure 6B) were expressed on cervical macrophages from PTB mice compared to normal pregnant controls. The expression of M1-associated molecules was significantly decreased and the expression of M2-associated molecules was significantly increased on cervical macrophages from nicotine treated compared to untreated PTB mice. The effects of nicotine were reversed by treatment with $\alpha$-BGT. These data suggest that nicotine could induce polarization of cervical macrophages to an anti-inflammatory M2 phenotype and create an antiinflammatory environment in the cervix.

Nicotine modulates LPS-induced phenotype-associated cytokines in the maternal cervix and amniotic fluid

To further investigate whether changes in macrophage polarization can influence the inflammatory microenvironment at the maternal-fetal interface, we detected mRNA expression of some key pro-inflammatory and anti-inflammatory genes in the cervix. LPS stimulation strongly increased the expression of TNF- $\alpha$, IL-1 $\beta$, CCL-2, CXCL10, CD86 and iNOS in the cervix of pregnant mice (Figure 8). In contrast, expression levels of antiinflammatory genes, including IL-10, Arg-1, CD163 and CD206, were lower after LPS stimulation. Nicotine treatment significantly suppressed the LPS-induced increase in proinflammatory gene expression in the cervix, but enhanced the levels of IL-10, Arg-1, CD163 and CD206. The $\alpha 7 n A c h R$ inhibitor ( $\alpha$-BGT) abolished the nicotine effects (Figure 8).

Amniotic fluid concentrations of cytokines were determined to reflect the fetal inflammatory response, which is also associated with poor pregnancy outcomes. IFN- $\gamma$, IL- 
1ß, IL-6, IL-13, CXCL10, CCL-2, CCL-5 and TNF- $\alpha$ levels were dramatically elevated in the amniotic fluid of pregnant mice injected with LPS; however, IL-10 and IL-4 levels were decreased (Figure 9). Nicotine significantly inhibited the LPS-induced changes. The beneficial effects of nicotine could be reversed by $\alpha$-BGT.

Activation of $\alpha 7 n A c h R$ by nicotine modulated down-stream signaling pathways related to cervical macrophage polarization in PTB mice

To elucidate the mechanisms by which $\alpha 7 n A c h R$ regulates cervical macrophage polarization in PTB, we investigated changes in the expression of the MAP kinases that mediate macrophage polarization in cervical tissues from PTB animals by Western blotting and double immunofluorescence staining. Results showed that the JNK and ERK1/2 signaling pathways regulated M1 macrophage polarization. Expression levels of phosphorylated JNK and ERK1/2 were significantly increased in cervical tissues from PTB group compared to normal pregnant controls. Nicotine treatment effectively inhibited the phosphorylation of JNK and ERK1/2 in PTB group (Figure 10A).

The pro-inflammatory NF-kB pathway mediates the anti-inflammatory effects of $\alpha 7 n A C h R$. To further examine the effects of $\alpha 7 n A c h R$ activation on the NF-kB pathway, we detected its expression and nuclear translocation in macrophages by Western blotting and double immunofluorescence staining. The expression of NF-kB was profoundly increased in cervical tissues from PTB mice compared to normal pregnant controls.

Immunofluorescence images showed a significant increase in NF-kB nuclear translocation in cervical macrophages from PTB mice. $\alpha 7 n A c h R$ activation decreased NF-kB activity (Figure 10B).

We also investigated the signaling pathways such as PI3K/AKT, which is responsible for the activation of M2 macrophages. Phosphorylation of JAK2, STAT3, and AKT was downregulated in cervical tissues from PTB mice compared to normal pregnant controls (Figure 10A), suggesting that activation of JAK2/SAT3 and PI3K/AKT was inhibited in cervical tissues from PTB mice. Nicotine treatment increased the phosphorylation of JAK2, STAT3, and AKT in cervical tissues from PTB mice (Figure 10A). 
The nicotine-induced modulation of JNK, ERK1/2, NF-KB nuclear translocation in cervical macrophages, JAK2/STAT3 and PI3K/AKT was rescued by treatment with $\alpha$-BGT, which inhibited $\alpha 7 \mathrm{nAChR}$ activity in cervical macrophages (Figure 10A and 10B).

\section{Discussion}

We previously found that changes in $\mathrm{a} 7 \mathrm{nAChR}$ activity participated in cervical ripening in normal pregnancy [31]. The present study further showed that a7nAChR expression on cervical macrophages decreased, the number of M1 macrophages increased, and collagen content decreased in PTB patients and a pregnant mouse model of inflammatory responses induced by LPS compared to normal pregnant controls. Activation of a7nAchR by nicotine rescued LPS-induced adverse pregnancy and neonatal outcomes, prevented a decrease in collagen content, increased polarization of cervical macrophages toward the anti-inflammatory M2 phenotype and decreased the inflammatory responses at maternal-fetal interface; all nicotine-induced effects were reversed by the a7nAChR antagonist a-BGT. Our studies reveal that the mechanisms underlying the inhibitory role of nicotine on cervical ripening in pregnant mice with systemic inflammation include reducing collagen degradation and cervical inflammation by inhibiting conversion of M2 macrophages to M1 macrophages.

a7nAChR is a primary receptor of the cholinergic anti-inflammatory pathway that exerts its antiinflammatory effects by decreasing the production of TNF- $a$ and other cytokines from macrophages [32]. In normal pregnant murine, we have shown that a7nAChR expression on cervical macrophages and cervical collagen content decreased, and the number of M1 macrophages and expression of M1 macrophage biomarkers increased from mid- to late pregnancy [31], while nicotine exposure prolonged gestation and inhibited cervical ripening [33]. Further, in pregnant animals administered LPS, we found that nicotine exerted anti-inflammatory effects by decreasing LPS-induced increases in serum TNF- $a$ and IL-6 concentrations, and rescued fetuses [26]. In pregnant women with threatened preterm labor, serum levels of inflammatory cytokines such as TNF-a and interleukin-10 (IL-10) are increased compared to controls without threatened preterm labor [34]. In the present study, the expression of TNF- $a$ and IL-1 $\beta$ on macrophages in the cervix of pregnant mice with systemic inflammation induced by LPS was apparently enhanced compared to normal pregnant controls, while the expression of a7nAChR on macrophages and collagen content were reduced. Nicotine decreased the expression of inflammatory cytokines and increased the expression of a7nAChR and collagens in the cervix of PTB model, and the a7nAChR antagonist reversed the actions of nicotine. Taken together, these data indicate that the inflammatory response plays an important role in the premature initiation of labor, and that activation of a7nAChR can prevent premature cervical ripening, possibly by reducing the systemic and local cervical inflammatory response.

Evidence suggests that differential macrophage polarization is involved in tissue remodeling, as transformation of tissue macrophages from the M1 to M2 phenotype may decrease inflammation and inhibit collagen degradation. In a rat model of muscle injury, modulating macrophage phenotypes by 
decreasing the number of $\mathrm{M} 1$ macrophages and increasing the number of $\mathrm{M} 2$ macrophages potentiated an anti-inflammatory effect and promoted tissue repair [20]. In a mouse model of myocardial infarction, inducing M2 macrophage polarization reduced left ventricular adverse remodeling and improved cardiac function [19]. During pregnancy-related disorders such as miscarriages [35] and spontaneous preterm labor [36], the polarization pattern of decidua macrophages skewed toward M1. Such M1 polarization could result in an excessive pro-inflammatory response at the maternal-fetal interface, and interrupt the normal maintenance of pregnancy. In the present study, we showed a similar trend towards M1 polarization in macrophages in the cervix in PTB model. These findings corresponded to an increase in extracellular MMP-9 expression. Activation of a7nAchR impeded PTB-associated macrophage M1 polarization and inhibited the expression of MMP-9. These data suggest that M1 polarization promoted collagen degradation and premature cervical maturation in PTB. Regulating a7nAChR activity changed the macrophage polarization state and prevented cervical maturation.

Several signaling pathways such as JAK2/STAT3, PI3K-AKT and MAPKs ( $p J N K, p 38, p E R K$ ) may modulate macrophage-mediated inflammation [37-39]. An in vitro study in microglia showed that an a7nAChR agonist rescued LPS-inhibited activation of JAK2/STAT3 and PI3K/AKT [39]. In peritoneal macrophages, a7nAChR's anti-inflammatory actions were associated with activation of the transcription factor STAT3 [40]. In murine macrophage RAW264.7 cells, pro-inflammatory M1 polarization was reduced by suppressing LPS-induced activation of the MAP kinases, ERK1/2, p38 and JNK, and nuclear translocation of the NF-KB p65 subunit [41]. In the present study, nicotine reduced M1 polarization in cervical macrophages in PTB mice. Potential underlying mechanisms involved the activation of JAK2/STAT3 and PI3K/AKT, and inhibition of pJNK and nuclear translocation of the NF-KB p65 subunit. These data suggest that the signaling pathways related to macrophage polarization vary in different tissues and pathological conditions. Other factors, such as microRNAs, may mediate the effects of a7nAChR on macrophage polarization $[42,43]$ and should be investigated in future studies.

\section{Conclusions}

In summary, our results indicate that in vivo activation of a7nAChR by nicotine on cervical macrophages in PTB model stimulates M2 macrophage polarization, suppresses the release of MMP-9 to prevent an excessive reduction in cervical collagen content and cervical ripening. Moreover, nicotine treatment decreased fetal inflammation and the number of newborn deaths. These data provide a better understanding of the protective role of nicotine in preterm labor and highlight the potential of nicotine as a therapeutic agent for preterm labor.

\section{Declarations}

\section{Author contributions}

Xinjia Han and Jinying Yang conceived and designed the experiments; Xinjia Han, Baohua Lin, Wen Ding, Juanhua Chen, Tiansong Zhang and Yumian Lai performed the experiments; Xinjia Han, Baohua Lin, and 
Wen Ding analyzed the data; Xinjia Han and Jinying Yang wrote, revised, and commented on the paper. All authors have read and approved the final manuscript.

\section{Acknowledgements}

We thank Medjaden Bioscience Limited for assistance editing the manuscript.

\section{Competing interests}

The authors declare that they have no competing interests.

\section{Availability of data and materials}

The datasets used and/or analyzed in this study will be made available by the authors on reasonable request.

\section{Consent for publication}

This study consists of human data; all included patients provided written informed consent.

\section{Ethics approval and consent to participate}

The study on human data was approved by the Ethics Committee of the Guangzhou Women and Children Medical Center (No. 2018041701 and No. 201922200). All animal protocols were approved by the Committee on the Ethics of Animal Experiments of Guangzhou Medical University (Permit Number: 2012-50) and in

accordance with the NIH Guidelines (NIH Publications No. 8023, revised 1978) for the Care and Use of Laboratory Animals.

\section{Funding}

This study was supported by the National Natural Science Foundation of China (81971417, and 81901568), the Guangdong Health and Family Planning Commission Project (A2017332), the Hospital 
fund project from Guangzhou Women and Children's Medical Center (Pre-NSFC-2016-001), and the Guangzhou Science and Technology Project (2016A020218001).

\section{References}

1. Hanley M, Sayres L, Reiff ES, Wood A, Grotegut CA, Kuller JA. Tocolysis: A Review of the Literature. Obstet Gynecol Surv. 2019;74:50-5.

2. Dehaene I, Bergman L, Turtiainen P, Ridout A, Mol BW, Lorthe E. from the International Spontaneous Preterm birth Young Investigators g: Maintaining and repeating tocolysis: A reflection on evidence. Semin Perinatol. 2017;41:468-76.

3. Chawanpaiboon S, Laopaiboon M, Lumbiganon P, Sangkomkamhang US, Dowswell T. Terbutaline pump maintenance therapy after threatened preterm labour for reducing adverse neonatal outcomes. Cochrane Database Syst Rev 2014:CD010800.

4. Gonzalez JM, Romero R, Girardi G. Comparison of the mechanisms responsible for cervical remodeling in preterm and term labor. J Reprod Immunol. 2013;97:112-9.

5. van Engelen E, Breeveld-Dwarkasing VN, Taverne MA, Everts ME, van der Weijden GC, Rutten VP. MMP-2 expression precedes the final ripening process of the bovine cervix. Mol Reprod Dev. 2008;75:1669-77.

6. Choi SJ, Jung KL, Oh SY, Kim JH, Roh CR. Cervicovaginal matrix metalloproteinase-9 and cervical ripening in human term parturition. Eur J Obstet Gynecol Reprod Biol. 2009;142:43-7.

7. Yonemoto H, Young CB, Ross JT, Guilbert LL, Fairclough RJ, Olson DM. Changes in matrix metalloproteinase (MMP)-2 and MMP-9 in the fetal amnion and chorion during gestation and at term and preterm labor. Placenta. 2006;27:669-77.

8. Litwiniuk M, Radowicka M, Krejner A, Wielgos M, Grzela T. The MMP-9/TIMP-1 imbalance and the reduced level of TGF-beta in the cervical area of amniotic membrane is a possible risk factor of PROM and premature labor - proof-of-concept study. Ginekol Pol. 2017;88:379-84.

9. Sundrani D, Narang A, Mehendale S, Joshi S, Chavan-Gautam P. Investigating the expression of MMPs and TIMPs in preterm placenta and role of $\mathrm{CpG}$ methylation in regulating MMP-9 expression. IUBMB Life. 2017;69:985-93.

10. Dubicke A, Fransson E, Centini G, Andersson E, Bystrom B, Malmstrom A, Petraglia F, SverremarkEkstrom E, Ekman-Ordeberg G. Pro-inflammatory and anti-inflammatory cytokines in human preterm and term cervical ripening. J Reprod Immunol. 2010;84:176-85.

11. Tornblom SA, Klimaviciute A, Bystrom B, Chromek M, Brauner A, Ekman-Ordeberg G. Non-infected preterm parturition is related to increased concentrations of IL-6, IL-8 and MCP-1 in human cervix. Reprod Biol Endocrinol. 2005;3:39.

12. Yellon SM. Contributions to the dynamics of cervix remodeling prior to term and preterm birth. Biol Reprod. 2017;96:13-23. 
13. Watari M, Watari H, DiSanto ME, Chacko S, Shi GP, Strauss JF 3. Pro-inflammatory cytokines induce expression of matrix-metabolizing enzymes in human cervical smooth muscle cells. Am J Pathol. 1999;154:1755-62. rd. .

14. Zhou C, Huang M, Xie L, Shen J, Xiao T, Wang R. IVIG inhibits TNF-alpha-induced MMP9 expression and activity in monocytes by suppressing NF-kappaB and P38 MAPK activation. Int J Clin Exp Pathol. 2015;8:15879-86.

15. Liang Y, Yang N, Pan G, Jin B, Wang S, Ji W. Elevated IL-33 promotes expression of MMP2 and MMP9 via activating STAT3 in alveolar macrophages during LPS-induced acute lung injury. Cell Mol Biol Lett. 2018;23:52.

16. Sica A, Erreni M, Allavena P, Porta C. Macrophage polarization in pathology. Cell Mol Life Sci. 2015;72:4111-26.

17. Murray PJ. Macrophage Polarization. Annu Rev Physiol. 2017;79:541-66.

18. Gindele JA, Mang S, Pairet N, Christ I, Gantner F, Schymeinsky J, Lamb DJ. Opposing effects of in vitro differentiated macrophages sub-type on epithelial wound healing. PLoS One. 2017;12:e0184386.

19. Kimura T, Tajiri K, Sato A, Sakai S, Wang Z, Yoshida T, Uede T, Hiroe M, Aonuma K, leda M, ImanakaYoshida K. Tenascin-C accelerates adverse ventricular remodelling after myocardial infarction by modulating macrophage polarization. Cardiovasc Res. 2019;115:614-24.

20. Souza NHC, Mesquita-Ferrari RA, Rodrigues M, da Silva DFT, Ribeiro BG, Alves AN, Garcia MP, Nunes FD, da Silva Junior EM, Franca CM, et al. Photobiomodulation and different macrophages phenotypes during muscle tissue repair. J Cell Mol Med. 2018;22:4922-34.

21. Dubicke A, Ekman-Ordeberg G, Mazurek P, Miller L, Yellon SM. Density of Stromal Cells and Macrophages Associated With Collagen Remodeling in the Human Cervix in Preterm and Term Birth. Reprod Sci. 2016;23:595-603.

22. Payne KJ, Clyde LA, Weldon AJ, Milford TA, Yellon SM. Residency and activation of myeloid cells during remodeling of the prepartum murine cervix. Biol Reprod. 2012;87:106.

23. Fujii T, Mashimo M, Moriwaki Y, Misawa H, Ono S, Horiguchi K, Kawashima K. Physiological functions of the cholinergic system in immune cells. J Pharmacol Sci. 2017;134:1-21.

24. Vang A, Clements RT, Chichger H, Kue N, Allawzi A, O'Connell K, Jeong EM, Dudley SC Jr, Sakhatskyy $P$, Lu Q, et al. Effect of alpha7 nicotinic acetylcholine receptor activation on cardiac fibroblasts: a mechanism underlying RV fibrosis associated with cigarette smoke exposure. Am J Physiol Lung Cell Mol Physiol. 2017;312:L748-59.

25. Sun P, Li L, Zhao C, Pan M, Qian Z, Su X. Deficiency of alpha7 nicotinic acetylcholine receptor attenuates bleomycin-induced lung fibrosis in mice. Mol Med. 2017;23:34-9.

26. Yang J, Shi SQ, Shi L, Fang D, Liu H, Garfield RE. Nicotine, an alpha7 nAChR agonist, reduces lipopolysaccharide-induced inflammatory responses and protects fetuses in pregnant rats. Am J Obstet Gynecol. 2014;211:538 e531-7. 
27. Arenas-Hernandez M, Romero R, St Louis D, Hassan SS, Kaye EB, Gomez-Lopez N. An imbalance between innate and adaptive immune cells at the maternal-fetal interface occurs prior to endotoxininduced preterm birth. Cell Mol Immunol. 2016;13:462-73.

28. Arenas-Hernandez M, Romero R, Xu Y, Panaitescu B, Garcia-Flores V, Miller D, Ahn H, Done B, Hassan SS, Hsu CD, et al. Effector and Activated T Cells Induce Preterm Labor and Birth That Is Prevented by Treatment with Progesterone. J Immunol. 2019;202:2585-608.

29. West KL, Proia AD, Puri PK. Fontana-Masson stain in fungal infections. J Am Acad Dermatol. 2017;77:1119-25.

30. Ji J, He L: Effect of Kangxianling Decoction on Expression of TGF-beta1/Smads and Extracellular Matrix Deposition. Evid Based Complement Alternat Med 2019, 2019:5813549.

31. Yang J, Lai Y, Chen J, Lin B, Zhou B, Han X. Changes in alpha7nAChR and macrophage polarization state participate in the regulation of cervical remodeling in pregnant rats. Biol Reprod 2019.

32. Wang H, Yu M, Ochani M, Amella CA, Tanovic M, Susarla S, Li JH, Wang H, Yang H, Ulloa L, et al. Nicotinic acetylcholine receptor alpha7 subunit is an essential regulator of inflammation. Nature. 2003;421:384-8.

33. Yang J, Shi SQ, Shi L, Liu H, Fang D, Garfield RE. Nicotine treatment prolongs gestation and inhibits cervical ripening in pregnant rats. Am J Obstet Gynecol. 2014;210:76 e71-7.

34. Herrera-Munoz A, Fernandez-Alonso AM, Fischer-Suarez N, Chedraui P, Perez-Lopez FR. Maternal serum cytokine levels in pregnancies complicated with threatened preterm labour. Gynecol Endocrinol. 2017;33:408-12.

35. Li Z, Zhao M, Li T, Zheng J, Liu X, Jiang Y, Zhang H, Hu X. Decidual Macrophage Functional Polarization during Abnormal Pregnancy due to Toxoplasma gondii: Role for LILRB4. Front Immunol. 2017;8:1013.

36. Xu Y, Romero R, Miller D, Kadam L, Mial TN, Plazyo O, Garcia-Flores V, Hassan SS, Xu Z, Tarca AL, et al. An M1-like Macrophage Polarization in Decidual Tissue during Spontaneous Preterm Labor That Is Attenuated by Rosiglitazone Treatment. J Immunol. 2016;196:2476-91.

37. Kawamata J, Shimohama S. Stimulating nicotinic receptors trigger multiple pathways attenuating cytotoxicity in models of Alzheimer's and Parkinson's diseases. J Alzheimers Dis. 2011;24(Suppl 2):95-109.

38. Patel H, Mclntire J, Ryan S, Dunah A, Loring R. Anti-inflammatory effects of astroglial alpha7 nicotinic acetylcholine receptors are mediated by inhibition of the NF-kappaB pathway and activation of the Nrf2 pathway. J Neuroinflammation. 2017;14:192.

39. Zhang Q, Lu Y, Bian H, Guo L, Zhu H. Activation of the alpha7 nicotinic receptor promotes lipopolysaccharide-induced conversion of M1 microglia to M2. Am J Transl Res. 2017;9:971-85.

40. de Jonge WJ, van der Zanden EP, The FO, Bijlsma MF, van Westerloo DJ, Bennink RJ, Berthoud HR, Uematsu S, Akira S, van den Wijngaard RM, Boeckxstaens GE. Stimulation of the vagus nerve attenuates macrophage activation by activating the Jak2-STAT3 signaling pathway. Nat Immunol. 2005;6:844-51. 
41. Luo D, Guo Y, Cheng Y, Zhao J, Wang Y, Rong J. Natural product celastrol suppressed macrophage M1 polarization against inflammation in diet-induced obese mice via regulating Nrf2/HO-1, MAP kinase and NF-kappaB pathways. Aging. 2017;9:2069-82.

42. O'Connell RM, Chaudhuri AA, Rao DS, Baltimore D. Inositol phosphatase SHIP1 is a primary target of miR-155. Proc Natl Acad Sci U S A. 2009;106:7113-8.

43. Taganov KD, Boldin MP, Chang KJ, Baltimore D. NF-kappaB-dependent induction of microRNA miR146 , an inhibitor targeted to signaling proteins of innate immune responses. Proc Natl Acad Sci U S A. 2006;103:12481-6.

\section{Figures}
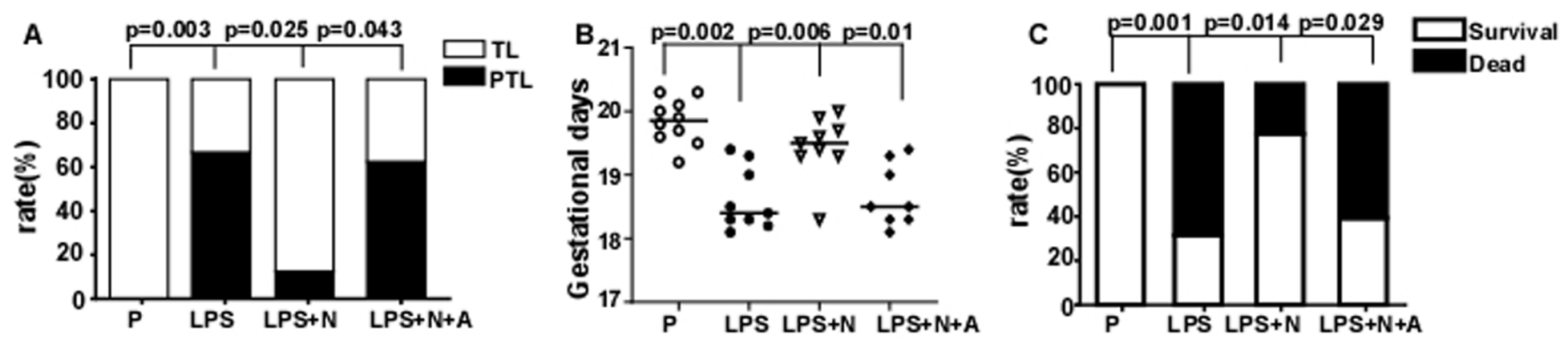

Figure 1

Effect of nicotine on the frequency of preterm labor $(A)$ and gestational age $(B)$ in pregnant mice injected with LPS, $n=8-10$ each. Effect of nicotine on the survival rate of pups born to pregnant mice injected with LPS (C), n=8-10 each. Data were analyzed with the Mann-Whitney $U$ test. 

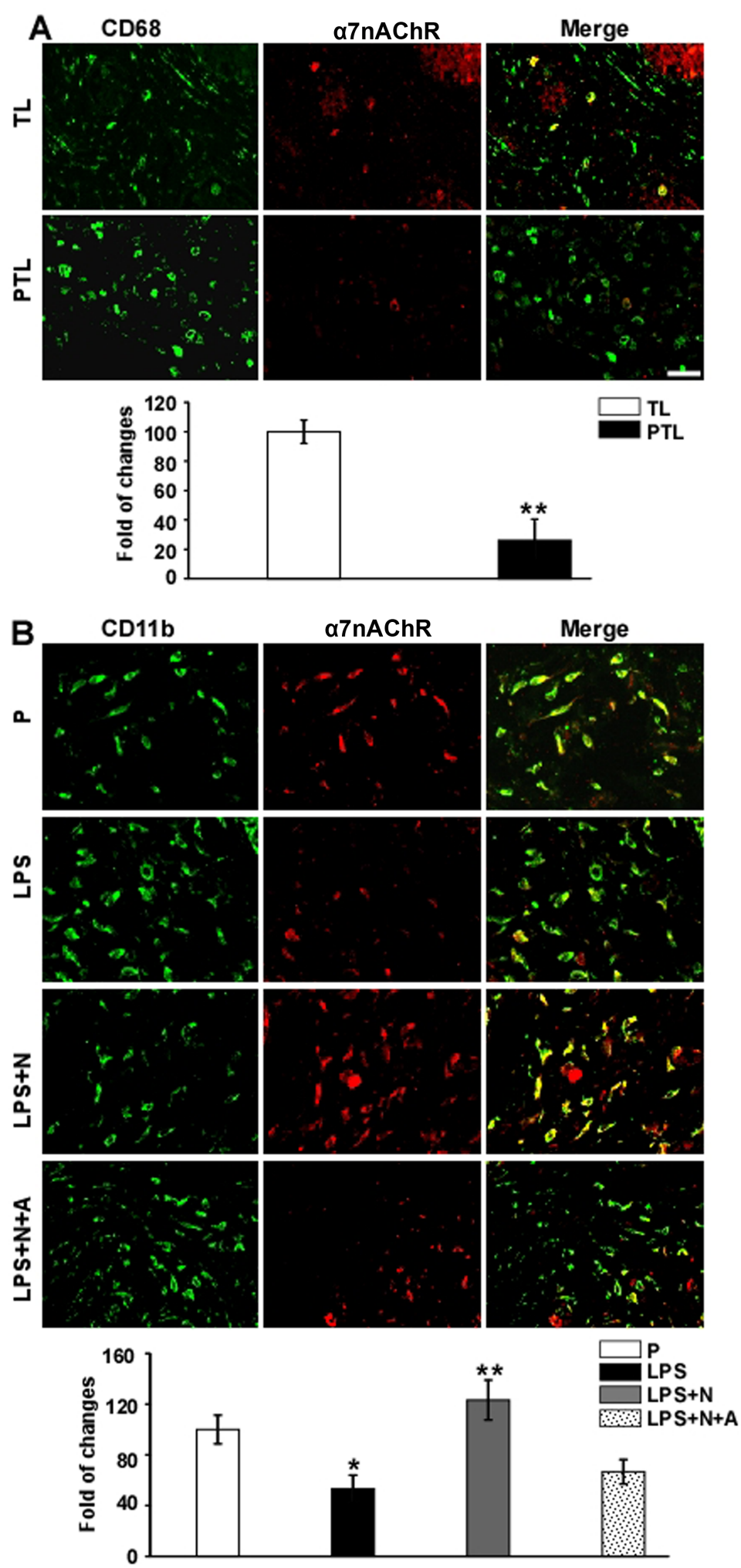

Figure 2

Decreased a7nAchR expression on cervical macrophages in PTB patients and mice. (A) Immunofluorescence staining and quantitative analysis showing the number of co-labeled $a 7 n A c h R$ and CD68 cervical macrophages from PTB patients and normal pregnant controls $(n=10-12)$. Data are presented as means \pm SEM. ${ }^{\star *} \mathrm{P}<0.01$. Scale bar $=30 \mu \mathrm{m}$. TL: term labor; PTL: preterm labor.

(B)Immunofluorescence staining and quantitative analysis showing the number of co-labeled $a 7 n A c h R$ 
and CD11b cervical macrophages from PTB mice and normal pregnant controls $(n=7)$. Data are presented as means \pm SEM. Statistical analyses were conducted using by one-way ANOVA, ${ }^{*} P<0.05, P T B$ vs. $P ; * * P<0.01, P T B+N$ vs. $P T B$ and $P T B+N+A$. Scale bar $=30 \mu m$.

A
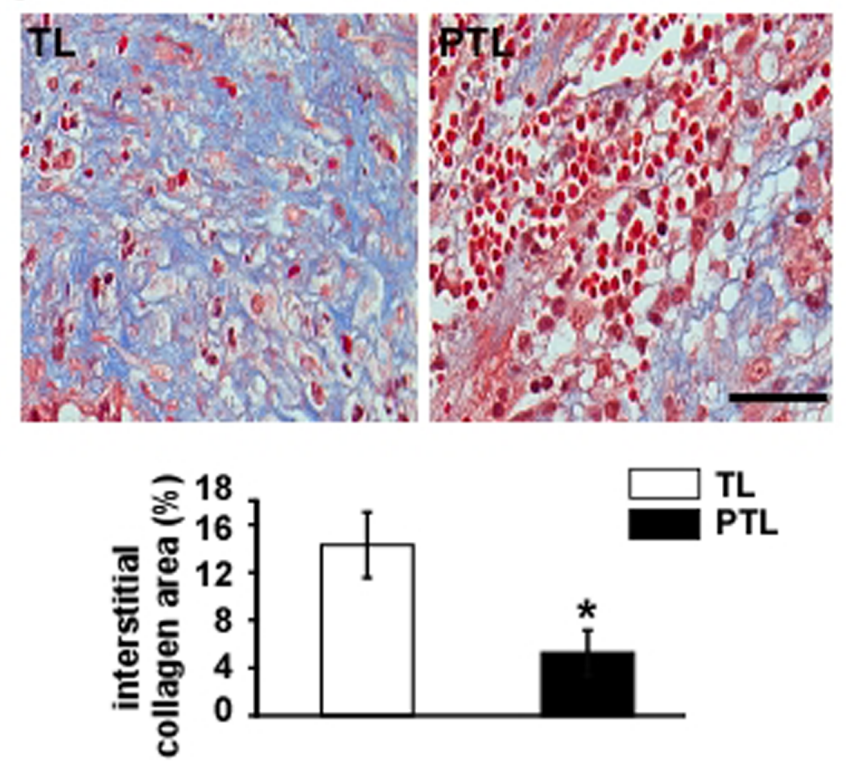

C
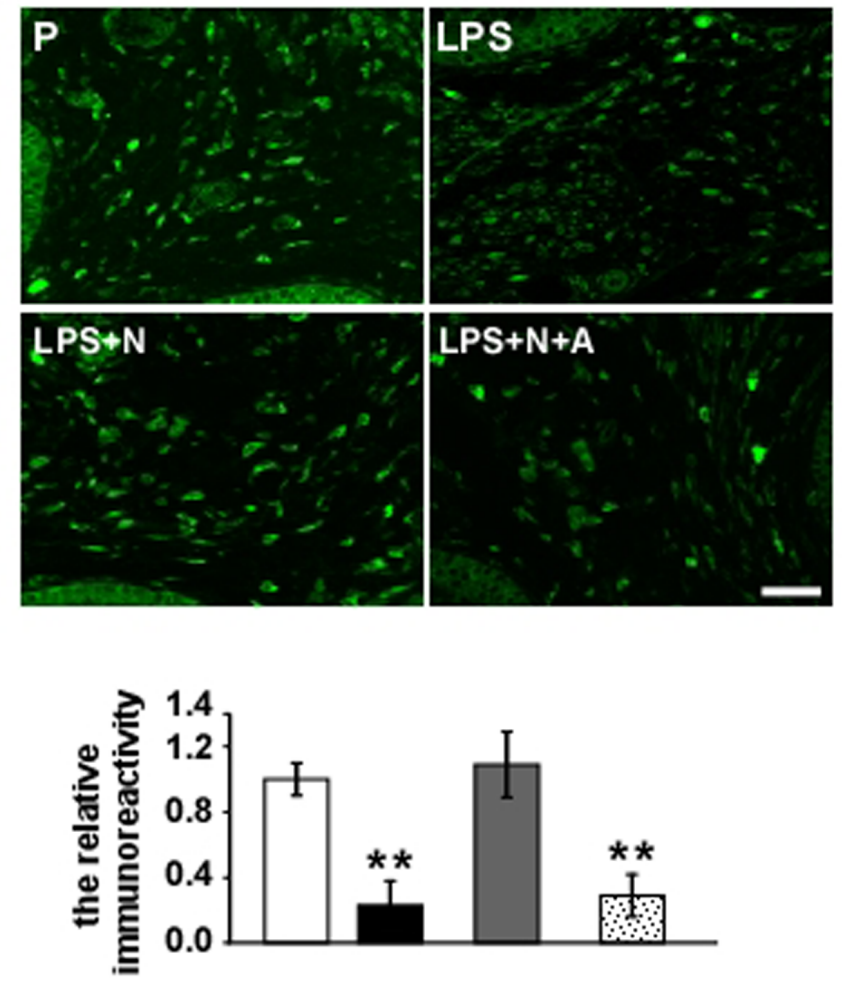

B
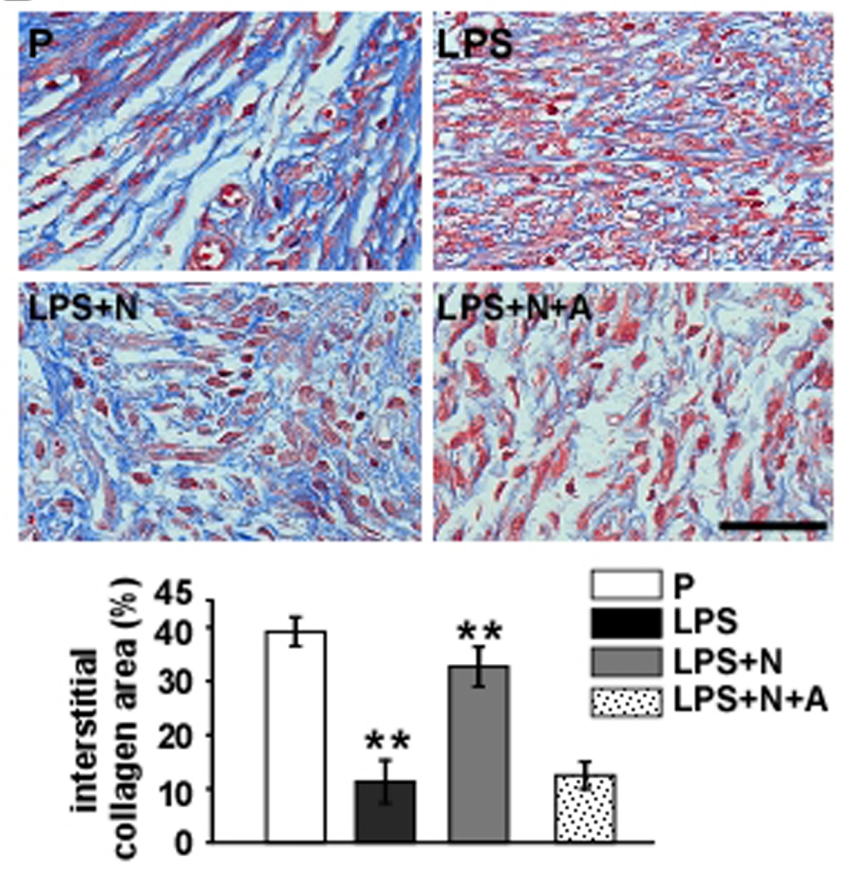

D collagen III
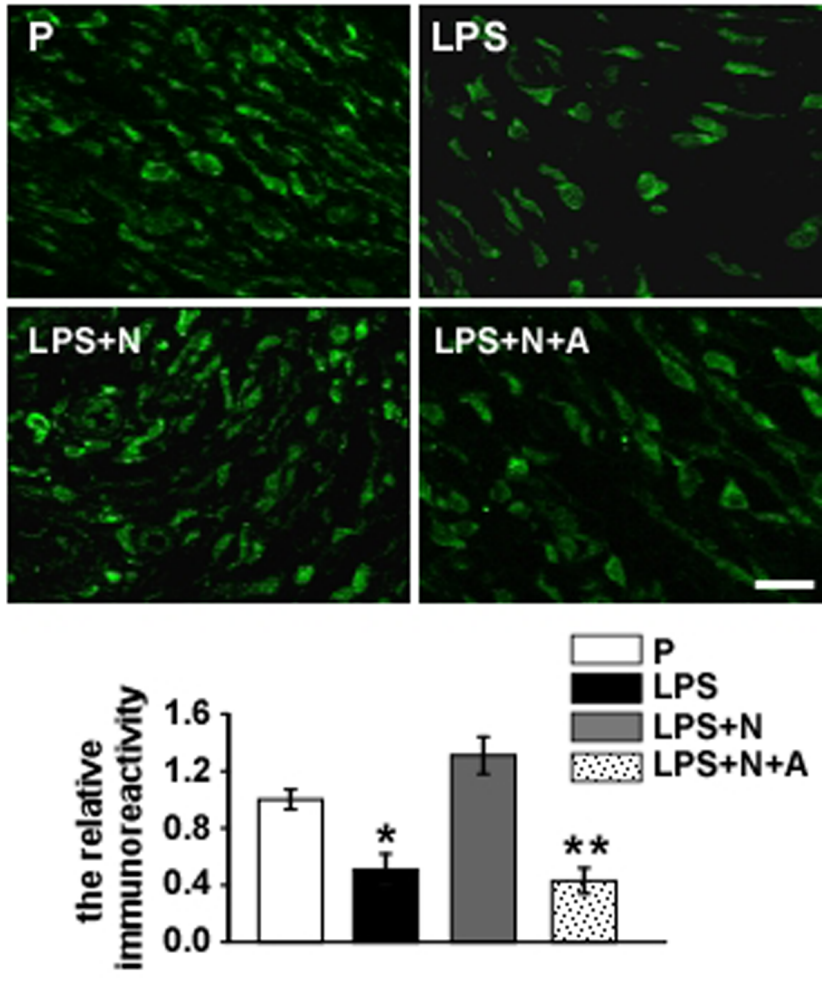

Figure 3

Nicotine treatment prevented the decrease in cervical collagen content in PTB mice (A) Histological staining (Masson's trichome) and quantitative analysis (percentage stained area) showing collagen 
content in cervical biopsies from PTB patients and normal pregnant controls $(n=10-12)$. Data are presented as means \pm SEM. ${ }^{*} P<0.05$. Scale bar $=50 \mu \mathrm{m}$. TL: term labor; PTL: preterm labor. $(B)$ Histological staining (Masson's trichome) and quantitative analysis (integrated optical density) showing collagen content in cervical tissues from PTB mice and normal pregnant controls $(n=6)$. Data are presented as mean \pm SEM. Statistical analyses were conducted using one-way ANOVA, ${ }^{\star * P}<0.01, P T B$ vs. $P ; * \star P<0.01, P T B+N$ vs. PTB and PTB+N+A. Scale bar $=50 \mu m$. (C) and (D) Immunofluorescence staining and quantitative analysis showing collagen type I and collagen type III content in cervical tissues from PTB mice and normal pregnant controls. Data are presented as mean \pm SEM. Statistical analyses were conducted using one-way ANOVA. (C) $* * P<0.01$, PTB vs. $\mathrm{P}$ and $\mathrm{PTB}+\mathrm{N} ;{ }^{*} \mathrm{P}<0.01, \mathrm{PTB}+\mathrm{N}+\mathrm{A}$ vs. $\mathrm{PTB}+\mathrm{N}$. Scale bar $=40 \mu \mathrm{m}$. (D) $* \mathrm{P}<0.05$, PTB vs. $\mathrm{P}$ and $\mathrm{PTB}+\mathrm{N} ; * * \mathrm{P}<0.01, \mathrm{PTB}+\mathrm{N}+\mathrm{A}$ vs. $\mathrm{PTB}+\mathrm{N}$. Scale bar $=40 \mu \mathrm{m}$.

A
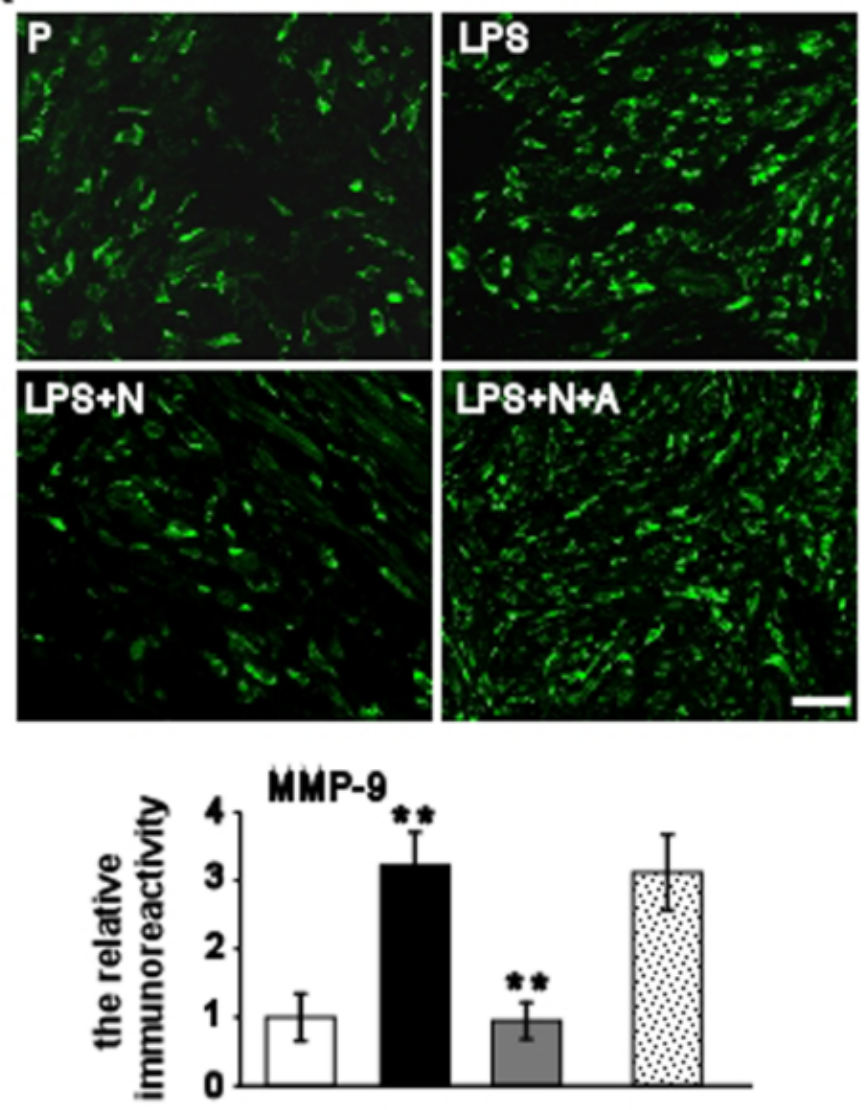

B
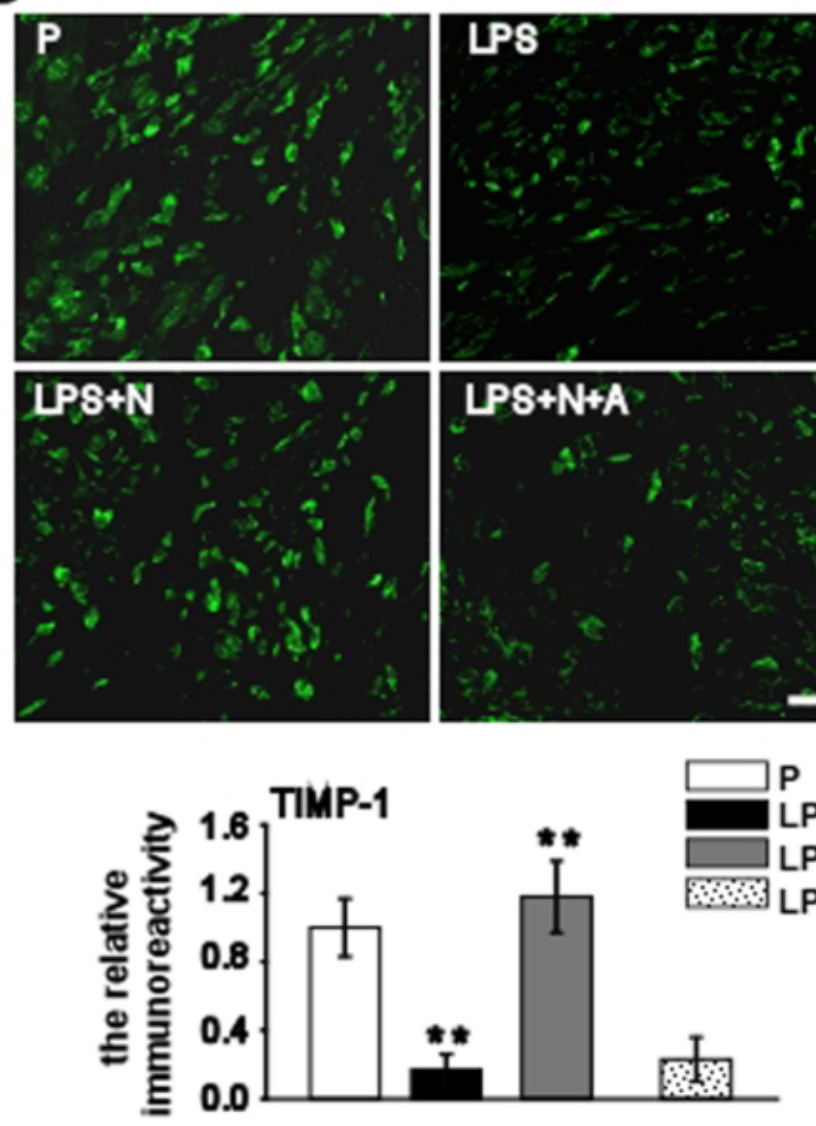
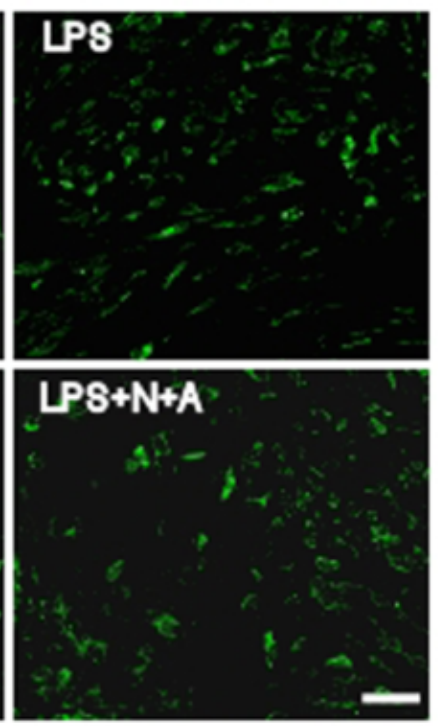
LPS LPS $+\mathrm{N}$ LPS $+\mathrm{N}+\mathrm{A}$

\section{Figure 4}

Nicotine treatment suppressed the release of matrix metalloproteinase-9 (MMP-9) in the cervical tissues of pregnant mice injected with LPS. Immunofluorescence staining and quantitative analysis (relative immunoreactivity) of MMP-9 (A) and its inhibitor TIMP-1 (B) in cervical tissues from PTB mice and normal pregnant controls $(n=7)$. Data are presented as mean \pm SEM. Statistical analyses were conducted by one-way ANOVA. (C) ${ }^{\star * P}<0.01$, PTB vs. $P ; * \star P<0.01, P T B+N$ vs PTB and PTB+N+A. Scale bar $=30 \mu m$. (D) ${ }^{\star * P}<<0.01$, PTB vs. $P$; ${ }^{*} P<0.01, P T B+N$ vs $P T B$ and $P T B+N+A$. Scale bar $=30 \mu \mathrm{m}$. 


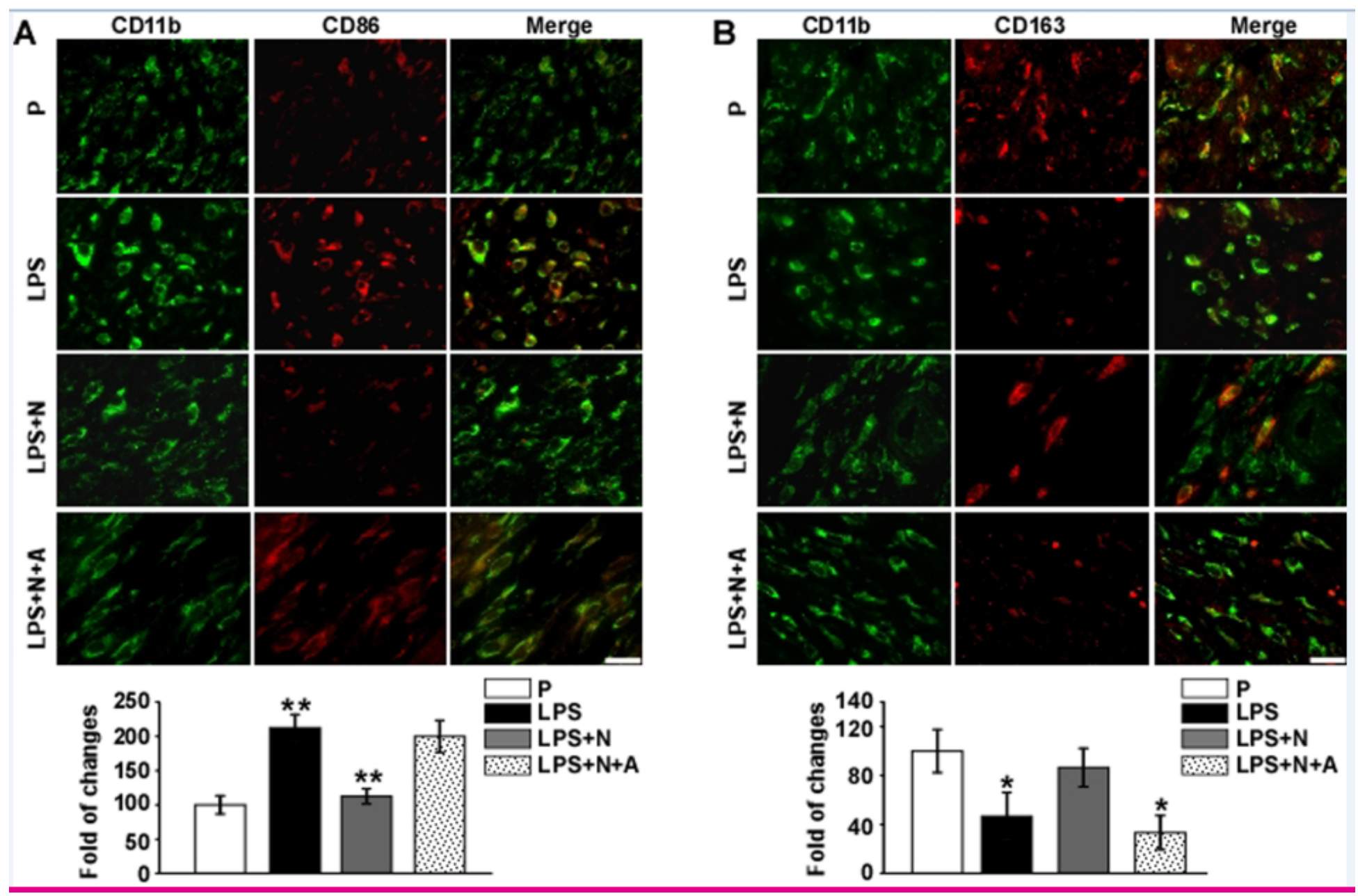

Figure 5

Nicotine treatment affected the differential expression of macrophage M1 (CD86) and M2 (CD163) biomarkers in the cervical tissues of PTB mice. (A) Double immunofluorescence staining (CD86 and CD11b) and quantitative analysis of CD86-positive (M1) cervical macrophages in PTB mice and normal pregnant controls. Data are presented as mean \pm SEM. Statistical analyses were conducted by one-way ANOVA, ${ }^{*} \mathrm{P}<0.01$, PTB vs. $\mathrm{P} ;{ }^{*} \mathrm{P}<0.01, \mathrm{PTB}+\mathrm{N}$ vs $\mathrm{PTB}$ and $\mathrm{PTB}+\mathrm{N}+\mathrm{A}$. Scale bar $=20 \mu \mathrm{m}$. (B) Double immunofluorescence staining (CD163 and CD11b) and quantitative analysis of CD163-positive (M2) cervical macrophages in PTB mice and normal pregnant controls $(n=7)$. Data are presented as mean \pm SEM. Statistical analyses were conducted by one-way ANOVA, ${ }^{*} \mathrm{P}<0.05$, PTB vs. $P$ and $P T B+N ;{ }^{*} P<0.05$, $\mathrm{PTB}+\mathrm{N}+\mathrm{A}$ vs. $\mathrm{PTB}+\mathrm{N}$. Scale bar $=20 \mu \mathrm{m}$. 


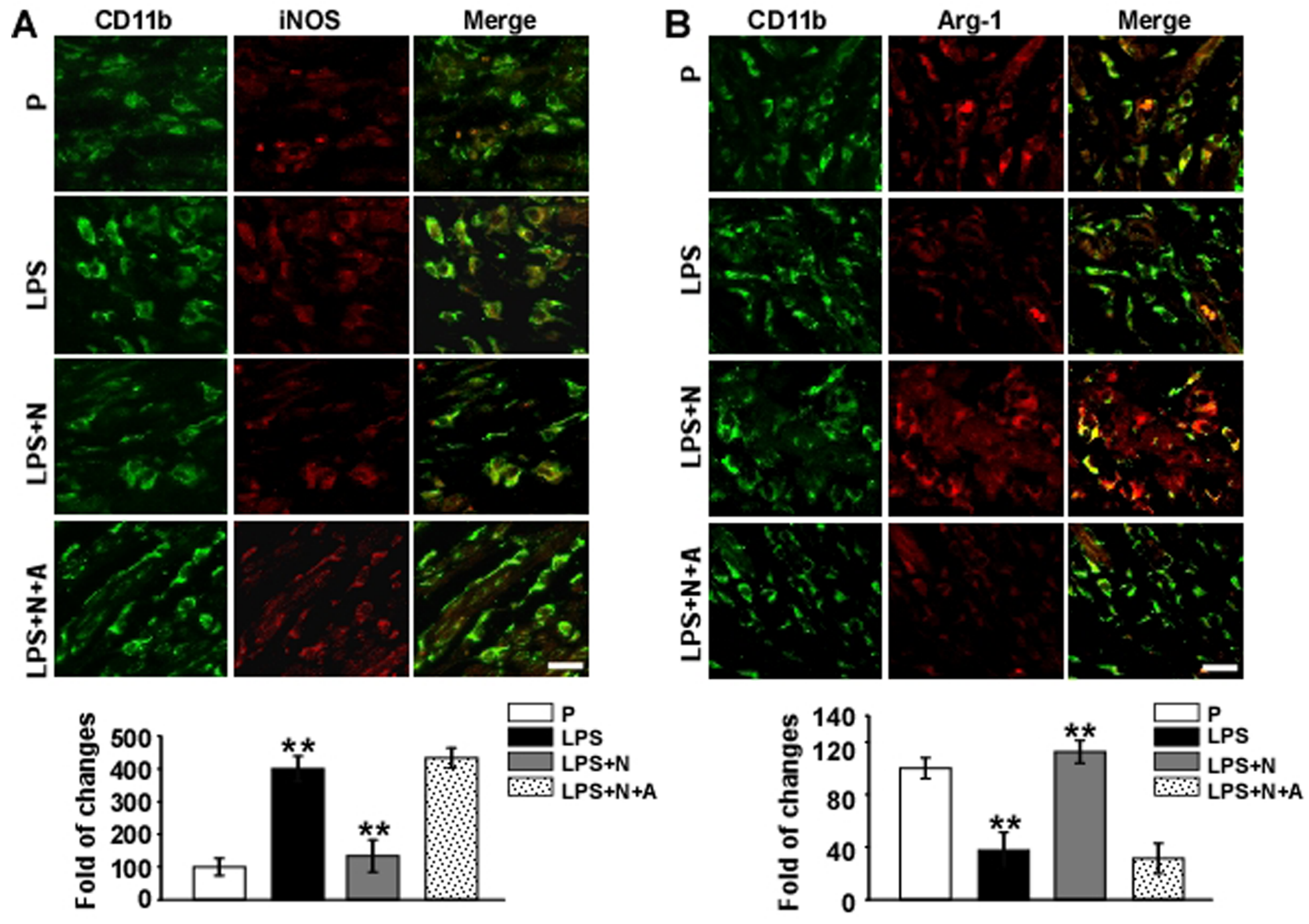

Figure 6

Nicotine treatment affected the differential expression of macrophage M1 (iNOS) andM2 (Arg-1) biomarkers in the cervical tissues of PTB mice. (A) Double immunofluorescence staining (iNOS and CD11b) and quantitative analysis of iNOS-positive (M1) cervical macrophages from PTB mice and normal pregnant controls $(n=7)$. Data are presented as mean \pm SEM. Statistical analyses were conducted by one-way ANOVA, ${ }^{*} \mathrm{P}<0.01$, PTB vs. $\mathrm{P} ;{ }^{*} \mathrm{P}<0.01, \mathrm{PTB}+\mathrm{N}$ vs. $\mathrm{PTB}$ and $\mathrm{PTB}+\mathrm{N}+\mathrm{A}$. Scale bar $=20 \mu \mathrm{m}$. (B) Double immunofluorescence staining (Arg-1 and CD11b) and quantitative analysis of Arg-1 positive (M2) cervical macrophages from PTB mice and normal pregnant controls $(n=7)$. $(n=7)$. Data are presented as mean \pm SEM. Statistical analyses were conducted by one-way ANOVA, ${ }^{*} P<0.01$, PTB vs. $P ;{ }^{\star *} P<0.01$, $\mathrm{PTB}+\mathrm{N}$ vs. $\mathrm{PTB}$ and $\mathrm{PTB}+\mathrm{N}+\mathrm{A}$. Scale bar $=20 \mu \mathrm{m}$. 


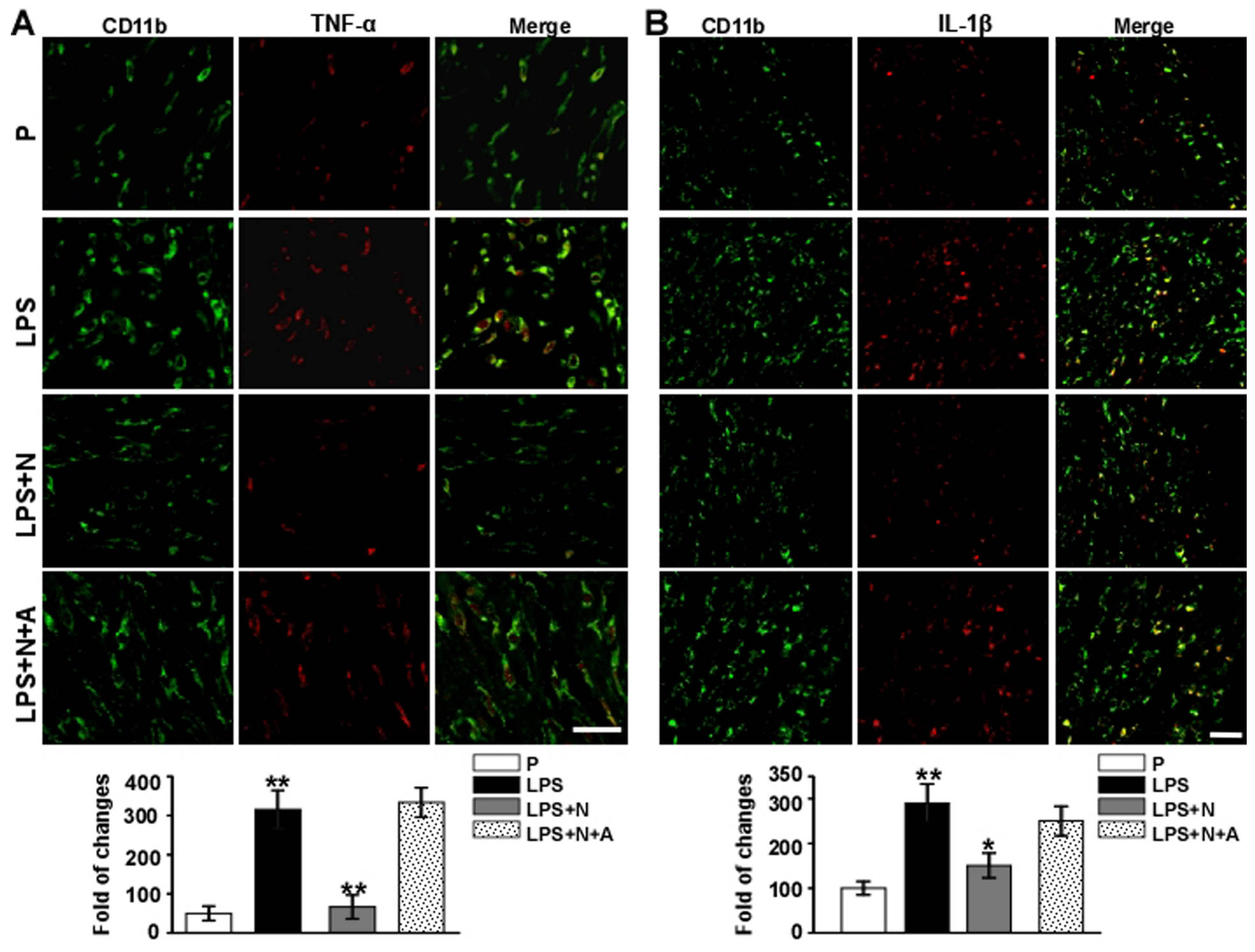

Figure 7

Nicotine treatment significantly reduced the expression of pro-inflammatory cytokines (TNF- $\alpha$ and IL-1 $\beta$ ) in the cervical tissues of PTB mice. Double immunofluorescence staining for CD11b and TNF- $a(A)$, CD11b and IL-1 $\beta$ (B); quantitative analysis showed changes in TNF- $a$ and IL-1 $\beta$ positive cervical macrophages $(n=7)$. Data are presented as mean \pm SEM. Statistical analyses were conducted by one-way ANOVA. (A) ${ }^{\star *} P<0.01$, PTB vs. $P ; * \star P<0.01, P T B+N$ vs $P T B$ and $P T B+N+A$. Scale bar $=25 \mu m$. (B) $* \star P<0.01$, PTB vs. $P ;{ }^{*}<<0.05, P T B+N$ vs $P T B$ and $P T B+N+A$. Scale bar $=25 \mu \mathrm{m}$. (C) ${ }^{\star} P<<0.01$, PTB vs. $P ; * \star P<0.01$, $\mathrm{PTB}+\mathrm{N}$ vs. $\mathrm{PTB}$ and $\mathrm{PTB}+\mathrm{N}+\mathrm{A}$. Scale bar $=25 \mu \mathrm{m}$. 

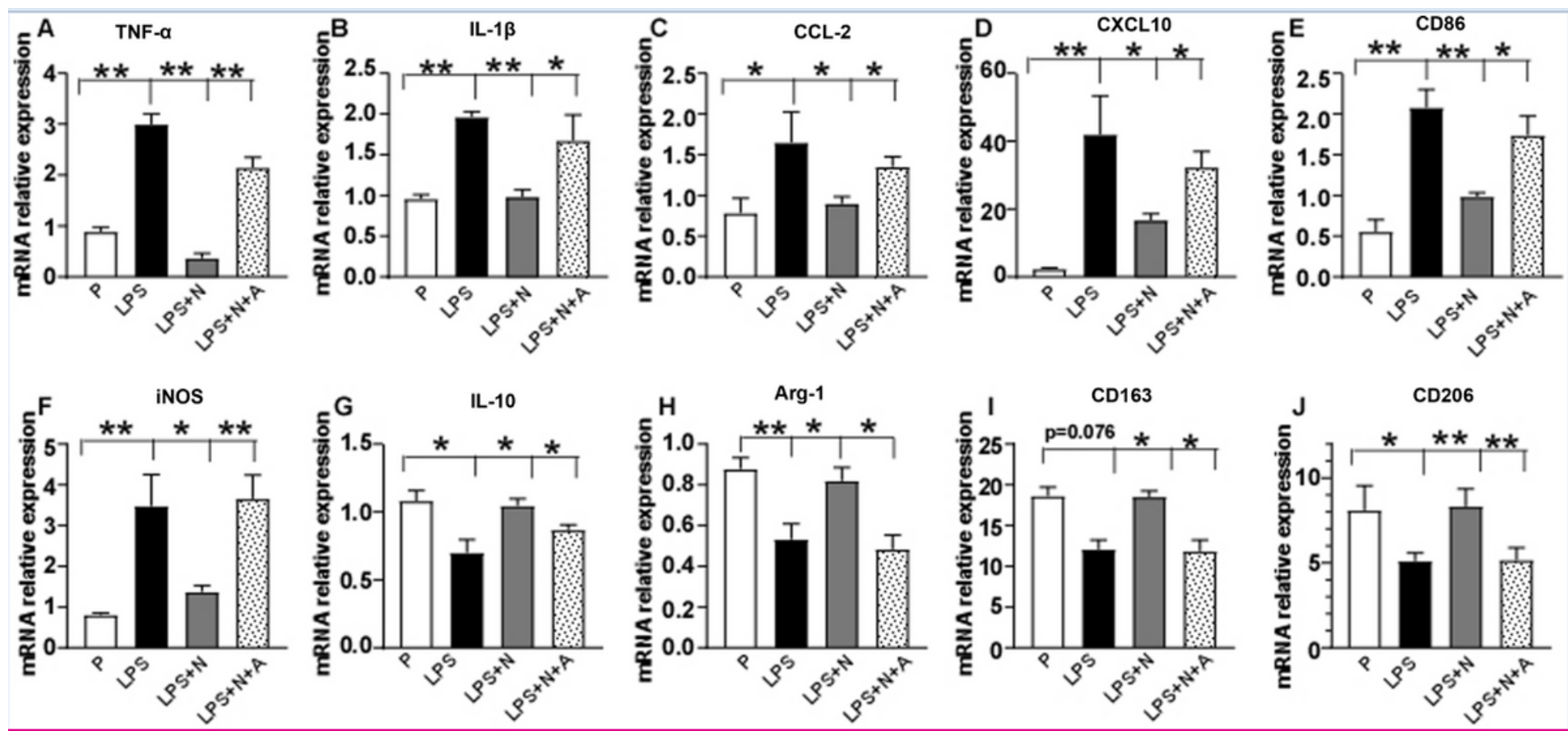

Figure 8

The effect of nicotine on the mRNA expression of pro-inflammatory cytokines, including TNF- $a(A)$, IL-1 $\beta$ (B), CCL-2 (C), CXCL10 (D), CD86 (E), iNOS (F) and anti-inflammatory cytokines including IL-10 (G), Arg$1(\mathrm{H}), \mathrm{CD} 163(\mathrm{I})$ and $\mathrm{CD} 206(\mathrm{~J})$ in the cervix of pregnant mice injected with LPS. $n=5$ each. ${ }^{*}<0.05$, $\star * P<0.01, * * * P<0.001$.

A IFN-Y

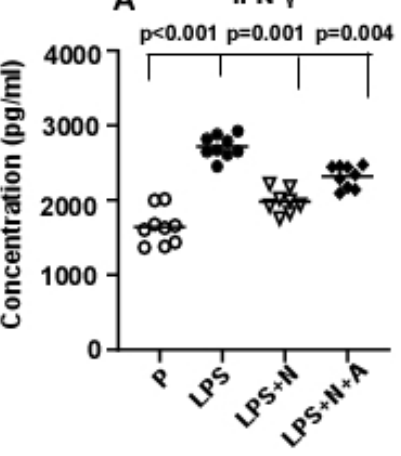

IL-13

$F_{p<0.001 p=0.001 p=0.027}$

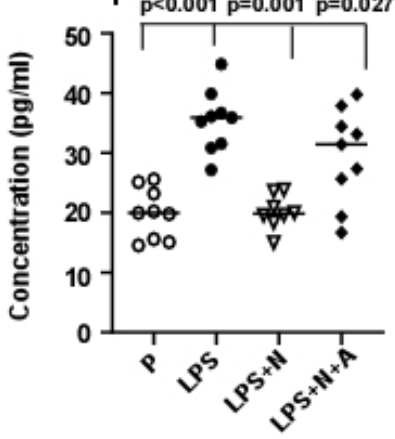

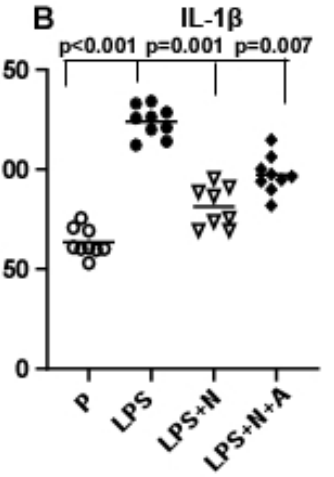

CXCL10

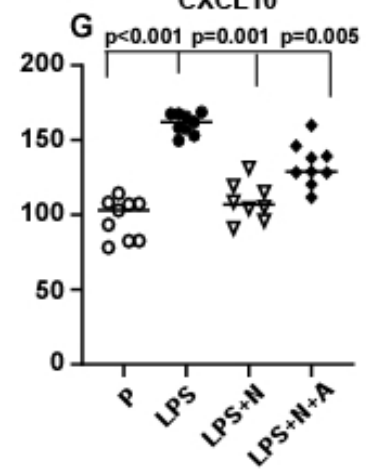

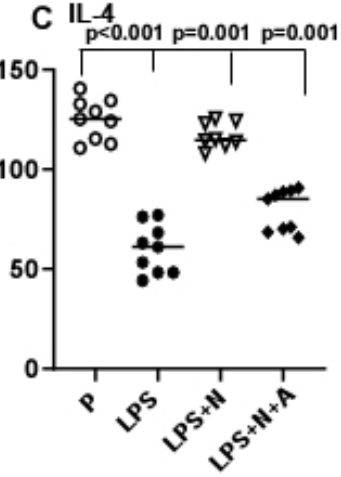

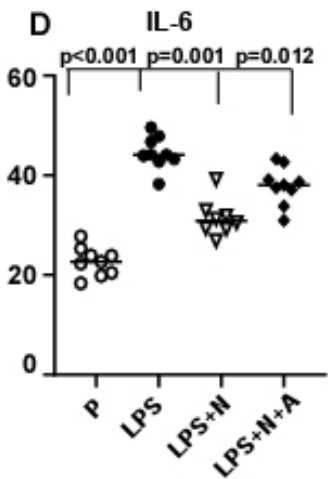

CCL-2

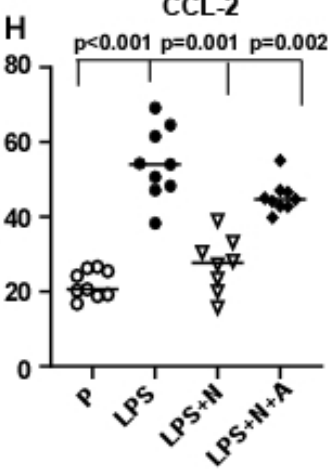

CCL-5

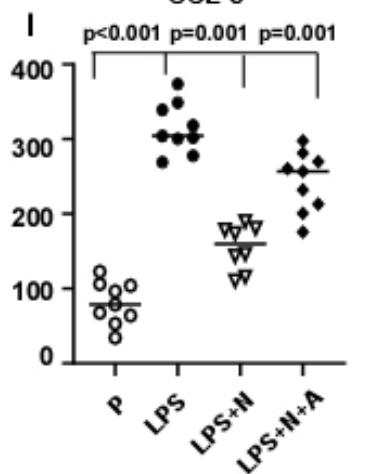

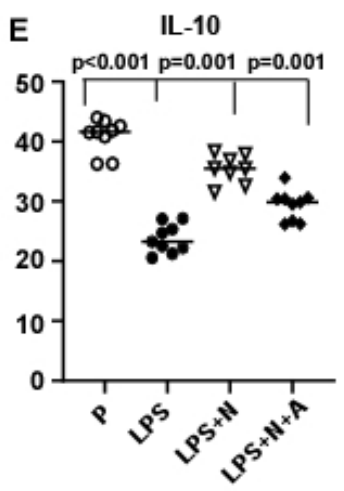

J $p=0.002 p=0.007 p=0.034$

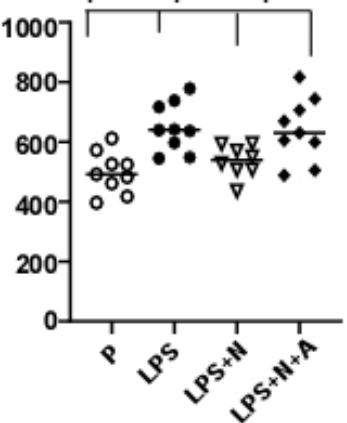

Figure 9 
The effects of nicotine on the LPS-induced secretion of cytokines in amniotic fluid from pregnant mice with or without $a-B G T$. The scatter plots demonstrated changes in the concentrations of cytokines in the amniotic fluid, including IFN- $\gamma(A), I L-1 \beta(B)$, IL-6 (C), IL-13 (D), CXCL10 (E), CCL-2 (F), CCL-5 (G) and TNF-a $(\mathrm{H}), \mathrm{IL}-10(\mathrm{I})$ and IL-4 $(\mathrm{J})$ in all experimental groups. $n=8-9$ each.

A
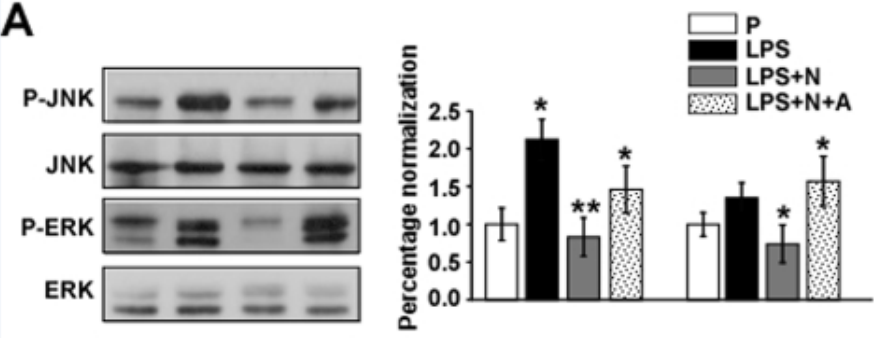

B
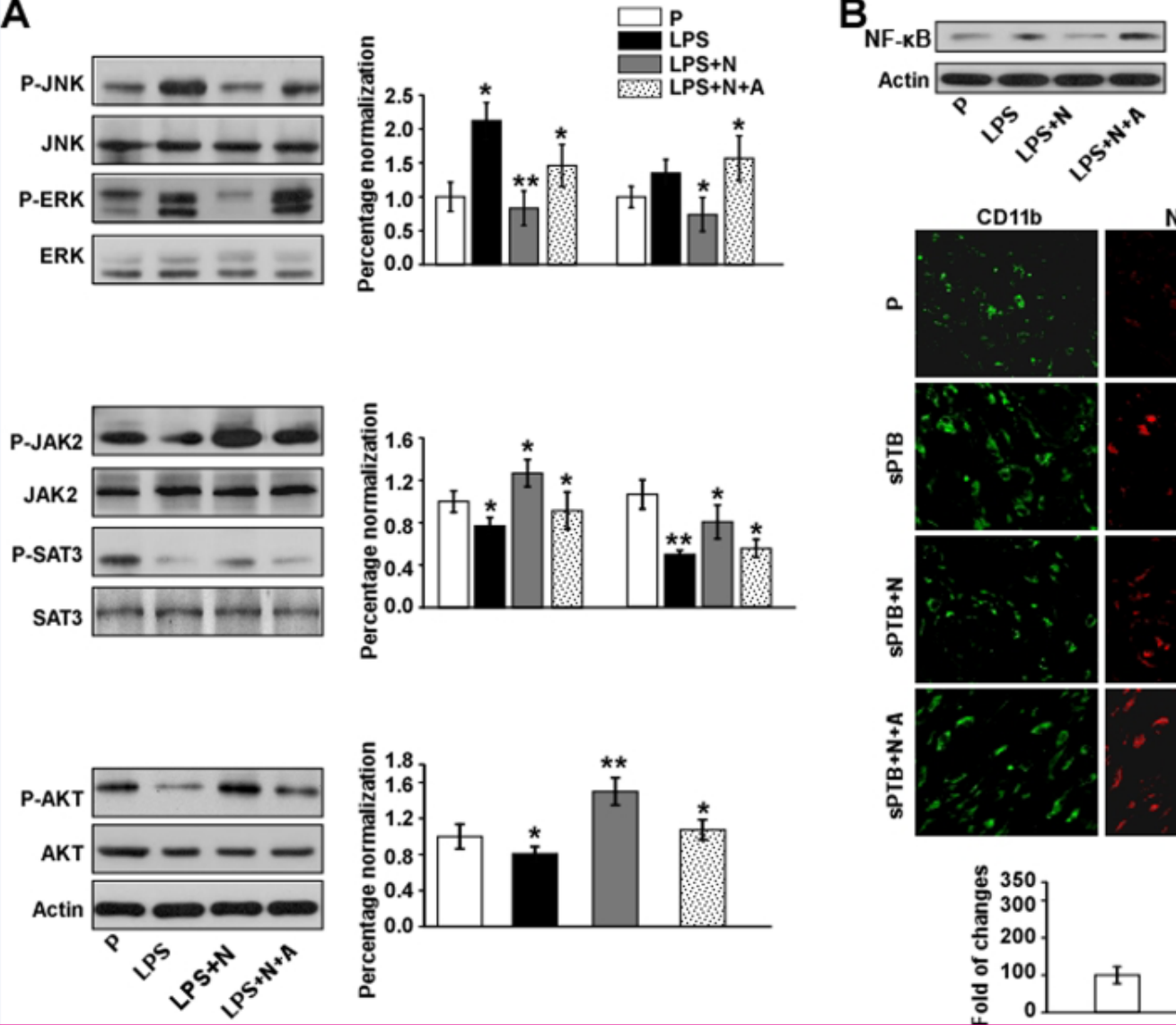
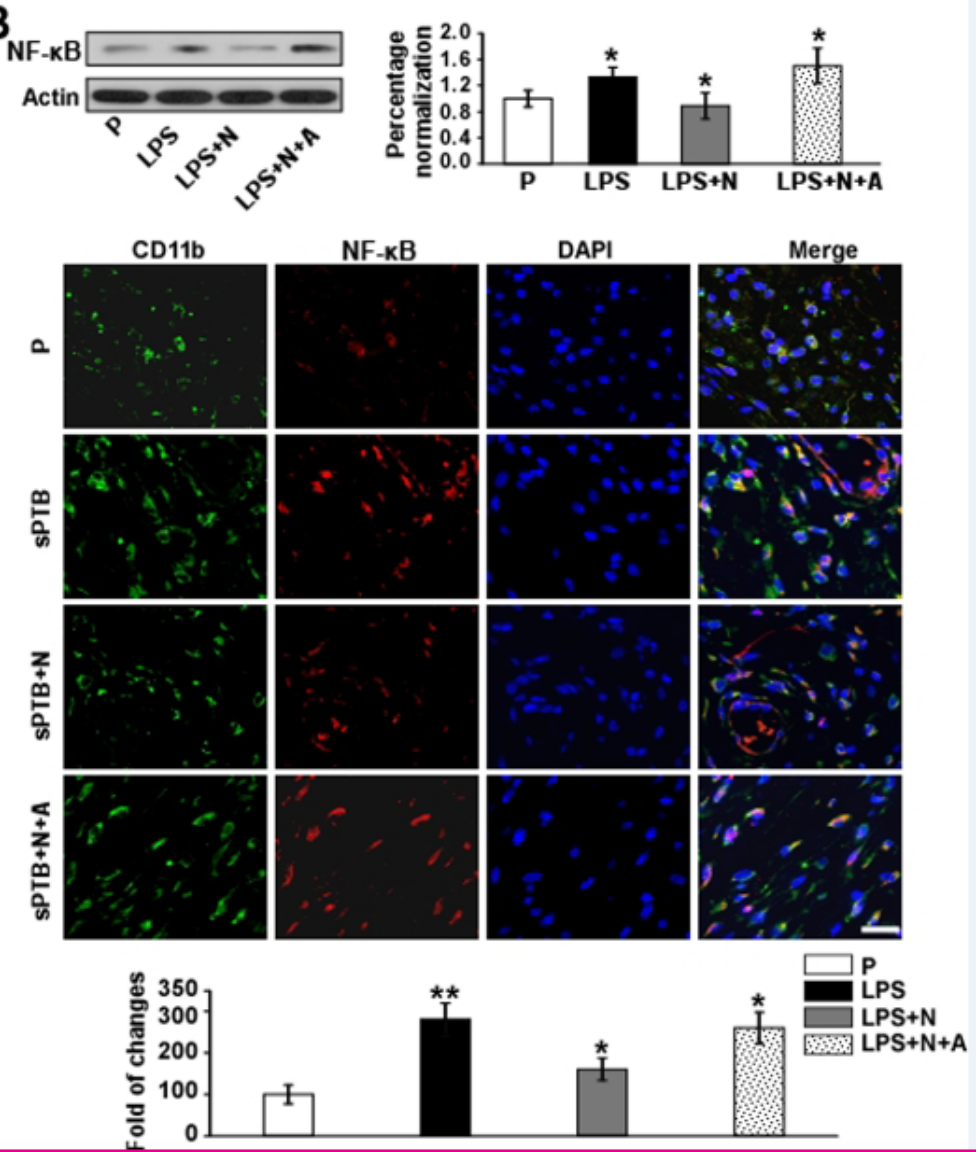

Figure 10

Nicotine inhibited LPS-induced activation of MAP kinases and NF-KB but reversed LPS-induced suppression of the JAK2/SAT3 and PI3K/AKT pathway in the cervical tissues of PTB mice. (A) Western blotting and quantitative analysis of JNK, p-JNK1/2, ERK1/2, p-ERK1/2, JAK2, p-JAK2, SAT3, p-SAT3, AKT, $p$-AKT protein levels in cervical tissues from PTB mice and normal pregnant controls $(n=5)$. Data are presented as mean \pm SEM. Statistical analyses were conducted by one-way ANOVA. $p-J N K / J N K:{ }^{*} P<0.05$, $P T B$ vs. $P ;{ }^{\star \star} P<0.01, P T B+N$ vs. $P T B ;{ }^{*}<0.05, P T B+N+A$ vs. $P T B+N . p-E R K 1 / 2 / E R K 1 / 2:{ }^{*}<<0.05, P T B+N$ vs. $\mathrm{PTB} ;{ }^{*} \mathrm{P}<0.05, \mathrm{PTB}+\mathrm{N}+A$ vs. $\mathrm{PTB}+\mathrm{N} . \mathrm{p}-J A K 2 / J A K 2:{ }^{*}<0.05, \mathrm{PTB}$ vs. $\mathrm{P} ;{ }^{*} \mathrm{P}<0.05, \mathrm{PTB}+\mathrm{N}$ vs. $\mathrm{PTB}$; ${ }^{\star} \mathrm{P}<0.05, \mathrm{PTB}+\mathrm{N}+A$ vs. $\mathrm{PTB}+\mathrm{N} . \mathrm{p}-\mathrm{SAT} 3 / \mathrm{SAT} 3:{ }^{*} \mathrm{P}<0.01$, PTB vs. $\mathrm{P} ;{ }^{*} \mathrm{P}<0.05, \mathrm{PTB}+\mathrm{N}$ vs. $\mathrm{PTB} ;{ }^{*} \mathrm{P}<0.05$, $\mathrm{PTB}+\mathrm{N}+\mathrm{A}$ vs. $\mathrm{PTB}+\mathrm{N}$. $\mathrm{p}-\mathrm{AKT} / \mathrm{AKT}$ : ${ }^{*} \mathrm{P}<0.05, \mathrm{PTB}$ vs. $\mathrm{P} ;{ }^{*} \mathrm{*}<0.01, \mathrm{PTB}+\mathrm{N}$ vs. $\mathrm{PTB} ;{ }^{*} \mathrm{P}<0.05, \mathrm{PTB}+\mathrm{N}+\mathrm{A}$ vs. $\mathrm{PTB}+\mathrm{N}$.(B) Western blotting and quantitative analysis of NF-KB protein levels in cervical tissues from PTB mice and normal pregnant controls $(n=5)$. ${ }^{*}<0.05$, PTB vs. $P$; ${ }^{*}<<0.05, P T B+N$ vs. $P T B ;{ }^{*}<<0.05$, $\mathrm{PTB}+\mathrm{N}+\mathrm{A}$ vs. $\mathrm{PTB}+\mathrm{N}$. Double immunofluorescence staining and quantitative analysis of NF-KB positive cervical macrophages and nuclear translocation of NF-KB (changes in the number of macrophages with NF-KB localized in the nuclei) in cervical macrophages from PTB mice and normal pregnant controls 
$(n=7)$. Data are presented as mean \pm SEM. Statistical analyses were conducted by one-way ANOVA, ${ }^{\star} \times P<0.01$, PTB vs. $P ;{ }^{*}<<0.05, P T B+N$ vs. $P T B ;{ }^{*}<<0.05, P T B+N+A$ vs. $P T B+N$. Scale bar $=25 \mu \mathrm{m}$. 